TRANSACTIONS OF THE

AMERICAN MATHEMATICAL SOCIETY

Volume 364, Number 8, August 2012, Pages 4085-4106

S 0002-9947(2012)05434-1

Article electronically published on March 28, 2012

\title{
FORMULAS FOR THE MULTIPLICITY OF GRADED ALGEBRAS
}

\author{
YU XIE
}

\begin{abstract}
Let $R$ be a standard graded Noetherian algebra over an Artinian local ring. Motivated by the work of Achilles and Manaresi in intersection theory, we first express the multiplicity of $R$ by means of local $j$-multiplicities of various hyperplane sections. When applied to a homogeneous inclusion $A \subseteq B$ of standard graded Noetherian algebras over an Artinian local ring, this formula yields the multiplicity of $A$ in terms of that of $B$ and of local $j$-multiplicities of hyperplane sections along $\operatorname{Proj}(B)$. Our formulas can be used to find the multiplicity of special fiber rings and to obtain the degree of dual varieties for any hypersurface. In particular, it gives a generalization of Teissier's Plücker formula to hypersurfaces with non-isolated singularities. Our work generalizes results by Simis, Ulrich and Vasconcelos on homogeneous embeddings of graded algebras.
\end{abstract}

\section{INTRODUCTION}

Let $A=A_{0}\left[A_{1}\right] \subseteq B=B_{0}\left[B_{1}\right]$ be a homogeneous inclusion of standard graded Noetherian rings with $A_{0}=B_{0}$ and each Artinian local. The goal of this work is to give a formula for the multiplicity of $A$ in terms of the multiplicity of $B$ and of local multiplicities along $\operatorname{Proj}(B)$.

One of the main applications of this formula will be to the computation of the multiplicity of special fiber rings. Let $R$ be a standard graded Noetherian algebra of dimension $d$ over a field $k$ and $I$ an ideal of $R$ generated by forms of the same degree $\delta$. Let $\mathscr{R}(I)=\bigoplus_{j=0}^{\infty} I^{j}$ be the Rees algebra of $I$. The special fiber ring $k \otimes_{R} \mathscr{R}(I)$ describes the homogeneous coordinate ring of the image of the rational map induced by $I$. As a special case this construction yields homogeneous coordinate rings of Gauss images and of secant varieties. It is important to compute the multiplicity of the special fiber ring. For this goal, observe $k \otimes_{R} \mathscr{R}(I) \simeq k\left[I_{\delta}\right] \subseteq k\left[R_{\delta}\right]=R^{(\delta)}$, where $k\left[I_{\delta}\right]$ is the $k$-algebra generated by the forms in $I$ of degree $\delta$ and $R^{(\delta)}$ is the $\delta$-th Veronese subring of $R$. After rescaling the grading of both $k\left[I_{\delta}\right]$ and $R^{(\delta)}, k\left[I_{\delta}\right] \subseteq R^{(\delta)}$ is a homogeneous inclusion of standard graded $k$-algebras. If we could express $e\left(k\left[I_{\delta}\right]\right)$ in terms of $e\left(R^{(\delta)}\right)$ and local multiplicities along $\operatorname{Proj}\left(R^{(\delta)}\right)$, then we can compute $e\left(k\left[I_{\delta}\right]\right)$ using the multiplicities of $R$. Indeed, since the $i$-th homogeneous component of $R^{(\delta)}$ is the $\delta i$-th homogeneous component of $R$,

Received by the editors October 7, 2009 and, in revised form, July 26, 2010.

2010 Mathematics Subject Classification. Primary 13H15, 13A30; Secondary 14J70, 14B05.

Key words and phrases. $j$-multiplicity, associated graded ring, special fiber ring, dual variety, Plücker formula.

This paper is based on the author's Ph.D. thesis, written under the direction of Professor Bernd Ulrich. The author sincerely thanks Professor Ulrich for suggesting the problem and for advice and many helpful discussions.

(C)2012 American Mathematical Society Reverts to public domain 28 years from publication 
$e\left(R^{(\delta)}\right)=e(R) \cdot \delta^{d-1}$. Furthermore, as $\operatorname{Proj}\left(R^{(\delta)}\right) \simeq \operatorname{Proj}(R)$, the local multiplicities along $\operatorname{Proj}\left(R^{(\delta)}\right)$ do not change if we pass to the local multiplicities along $\operatorname{Proj}(R)$.

For $A \subseteq B$ as above, it is easy to see that $\operatorname{dim} A \leq \operatorname{dim} B$. If $\operatorname{dim} A=\operatorname{dim} B$, the first case to consider occurs when $\operatorname{dim} B / A_{1} B=0$, i.e., $B$ is integral over $A$. If we assume further that $A_{p}$ is reduced and $\operatorname{rank}_{A_{p}} B_{p}=r$ for every prime ideal $p$ in $A$ of dimension $d$, then $e(B)=r e(A)$. The questions are as follows: What happens when $\operatorname{dim} B / A_{1} B \geq 1$ ? How do we find those local multiplicities along $\operatorname{Proj}(B)$ ? In 2001, Simis, Ulrich and Vasconcelos gave some partial answers:

Theorem 1.1 ([17, 6.4]). Let $A \subseteq B$ be a homogeneous inclusion of standard graded Noetherian rings of the same dimension, with $A_{0}=B_{0}$ each Artinian local and $B$ equidimensional. Let $\mathfrak{P}=\{p \in \operatorname{Spec}(A) \mid \operatorname{dim} A / p=\operatorname{dim} A\}$ and assume that $A_{p}$ is reduced and $\operatorname{rank}_{A_{p}} B_{p}=r$ for every prime ideal $p \in \mathfrak{P}$. If $\operatorname{dim} B / A_{1} B=1$, then

$$
e(B)=r e(A)+\sum_{q \in \mathfrak{Q}} e_{A_{1} B_{q}}\left(B_{q}\right) e(B / q),
$$

where $\mathfrak{Q}=\left\{q \in V\left(A_{1} B\right) \cap \operatorname{Proj}(B) \mid \operatorname{dim} B / q=\operatorname{dim} B / A_{1} B\right\}$.

Here $e(A)$ and $e(B)$ are the usual multiplicities of $A$ and $B$. We use $e_{A_{1} B_{q}}\left(B_{q}\right)$ to denote the Hilbert-Samuel multiplicity of $B_{q}$ with respect to the ideal $A_{1} B_{q}$.

The assumption $\operatorname{dim} B / A_{1} B=1$ forces the prime ideals in the projective spectrum of $B$ containing the ideal $A_{1} B$ to be minimal over $A_{1} B$. Therefore, the ideal $A_{1} B_{q}$ is primary to the maximal ideal $q B_{q}$, for $q \in \mathfrak{Q}$, and one can use the HilbertSamuel multiplicity $e_{A_{1} B_{q}}\left(B_{q}\right)$. But when $\operatorname{dim} B / A_{1} B>1$, the sum will involve prime ideals which are not minimal over $A_{1} B$ and the Hilbert-Samuel multiplicity is not defined locally at those prime ideals. Thus for $\operatorname{dim} B / A_{1} B>1$, the authors of [17] showed only that the left-hand side of equation (1) is greater than the righthand side. In this theorem, the required local multiplicities along $\operatorname{Proj}(B)$ are just the Hilbert-Samuel multiplicities.

Later in 2007, Validashti improved this inequality when $\operatorname{dim} B / A_{1} B>1$ :

Proposition 1.2 (21, 5.9.4]). Let $A \subseteq B$ be a homogeneous inclusion of standard graded Noetherian rings of the same dimension, with $A_{0}=B_{0}$ each Artinian local and $B$ equidimensional. Let $\mathfrak{P}=\{p \in \operatorname{Spec}(A) \mid \operatorname{dim} A / p=\operatorname{dim} A\}$ and assume that $A_{p}$ is reduced and $\operatorname{rank}_{A_{p}} B_{p}=r$ for every prime ideal $p \in \mathfrak{P}$. Then

$$
e(B) \geq \operatorname{re}(A)+\sum_{q \in \mathfrak{Q}^{\prime}} j\left(A_{1} B_{q}\right) e(B / q),
$$

where $\mathfrak{Q}^{\prime}=\left\{q \in V\left(A_{1} B\right) \cap \operatorname{Proj}(B) \mid \ell\left(A_{1} B_{q}\right)=\operatorname{dim} B_{q}\right\}$.

Here $\ell\left(A_{1} B_{q}\right)$ is the analytic spread of the ideal $A_{1} B_{q}$ and $j\left(A_{1} B_{q}\right)$ is its $j$-multiplicity in the sense of Achilles and Manaresi [1, 2]. Observe the set $\mathfrak{Q}^{\prime}$ is finite (it is contained in the set of the centers of the Rees valuations of $A_{1} B$ ). But it may contain prime ideals $q$ which are not minimal over $A_{1} B$. Hence the $j$-multiplicity is used in inequality (2) to replace the Hilbert-Samuel multiplicity. Since $A_{1} B_{q}$ has maximal analytic spread, one has $j\left(A_{1} B_{q}\right) \neq 0$. Also if $q$ is minimal over $A_{1} B, j\left(A_{1} B_{q}\right)=e_{A_{1} B_{q}}\left(B_{q}\right)$. Thus when $\operatorname{dim} B / A_{1} B>1$, some terms are added to the right-hand side of equation (1) to make it closer to $e(B)$. But Validashti 21] also gave an example to show that inequality (2) can be strict. In 
Theorem 4.1, we give the extra terms on the right-hand side of inequality (2) required to yield an equality for arbitrary dimensions of $B / A_{1} B$. This solves the problem when $\operatorname{dim} A=\operatorname{dim} B$. In Theorem 4.9, we also provide a formula for the case when $\operatorname{dim} A<\operatorname{dim} B$. Thus we give a complete answer to the original question.

As mentioned before, these formulas can be used to find the multiplicity of the special fiber ring $k \otimes_{R} \mathscr{R}(I)$, where $I \subseteq R$ is an ideal generated by forms of the same degree in a standard graded $k$-algebra $R$. This yields an upper bound for the reduction number of $I$ with respect to any reduction. It also provides a formula for the degree of the image of the rational map induced by $I$. In particular, it gives the degree of dual varieties of hypersurfaces. The first formula relating the degree of the dual variety to the degree of the variety itself was given by Plücker in 1834 for complex plane curves with at most nodes and cusps as singularities [12, p. 857]. Later, in 1975 Teissier generalized Plücker's formula to hypersurfaces with at most isolated singularities [18, App. II]. In 1994, Kleiman generalized Teissier's Plücker formula to projective varieties with at most isolated singularities [11, Theorem 2]. In 1997, based on Kleiman's work, Thorup generalized the Plücker formula to projective varieties with arbitrary singularities using the Chow groups of the varieties 19. Both of their formulas assume the variety has non-deficient dual (i.e., the dual variety is a hypersurface). In that case the degree of the dual variety is called the class of the variety. Our formulas can be used to find the degree of the dual variety for any hypersurface without any restriction on its singularities and dual variety (i.e., we do not need the dual variety to be a hypersurface). In particular, it gives a generalization of Teissier's Plücker formula to hypersurfaces with arbitrary singularities. See Section 5 for these applications.

This paper is divided into five parts. In Section 2, we first fix notation which will be used throughout the paper. After that we define the $j$-multiplicity for ideals that have possibly non-maximal analytic spread and prove some facts about the $j$-multiplicity and (super-) reduction sequences. In Section 3, we express the multiplicity of a standard graded Noetherian algebra by means of local $j$-multiplicities of various hyperplane sections. This formula becomes simpler if the ideal $I$ satisfies condition $G_{t+1}$ and Artin-Nagata property $A N_{t-1}^{-}$, or if $I$ is a complete intersection for every $q \in V(I)$ with ht $q \leq t$, or is perfect of height two satisfying condition $G_{t+1}$, or is Gorenstein of height three satisfying condition $G_{t+1}$ (see Corollaries 3.3 and 3.5). In Section 4, we consider a homogeneous inclusion $A \subseteq B$ of standard graded Noetherian rings over an Artinian local ring and express the multiplicity of $A$ in terms of that of $B$ and of local $j$-multiplicities of hyperplane sections along $\operatorname{Proj}(B)$; this is done by applying the formulas obtained in Section 3. Finally in Section 5, we obtain the formulas for the multiplicity of special fiber rings and give some applications.

\section{Preliminaries}

In this section, we first fix notation and recall some basic concepts and results which will be used throughout the paper. Then we define the $j$-multiplicity for ideals that have possibly non-maximal analytic spread and prove some facts about the $j$-multiplicity and (super-) reduction sequences.

Throughout the paper, let $(R, m, k)$ be either a Noetherian local ring or a standard graded Noetherian algebra over an Artinian local ring $\left(R_{0}, m_{0}\right)$, where $m$ is either the maximal ideal or the homogeneous maximal ideal $\left(m_{0}, R_{1} R\right)$ of 
$R$ and $k=R / m$ is the residue field. Let $I$ be an ideal of $R$. Write $G=$ $\operatorname{gr}_{I}(R)=\bigoplus_{j=0}^{\infty} I^{j} / I^{j+1}$ for the associated graded ring of $R$ with respect to the ideal $I$ and $F=G / m G$ for the special fiber ring of this ideal. The analytic spread of $I$ is defined by $\ell(I)=\operatorname{dim} F$ and one has ht $I \leq \ell(I) \leq \operatorname{dim} R=\operatorname{dim} G$, where ht $I$ denotes the height of $I$.

If $I$ is an $m$-primary ideal of $R$, we write $e_{I}(R)$ for the Hilbert-Samuel multiplicity of $R$ with respect to $I$ and $e(R)$ for $e_{m}(R)$. If $I$ is not necessarily $m$-primary but has maximal analytic spread, i.e., $\ell(I)=\operatorname{dim} R$, one writes $j(I)$ for the $j$-multiplicity of $R$ with respect to $I$ (see [1, and Definition 2.1 and subsequent remarks below). Notice that if $I$ is $m$-primary, then $j(I)=e_{I}(R)$; see [1].

For an ideal $I$ and a submodule $N$ of an $R$-module $M$,

$$
N:_{M}\langle I\rangle=\bigcup_{i \geq 0}\left(N:_{M} I^{i}\right)=\left\{x \in M \mid I^{i} x \subseteq N \text { for some } i \in \mathbb{N}\right\} .
$$

An element $a \in I$ is said to be a filter-regular element with respect to $I$ if $0:_{R} a \subseteq$ $0:_{R}\langle I\rangle$. This is equivalent to saying that $a$ is not in any $q \in \operatorname{Ass}(R)$ such that $I \nsubseteq q$. A sequence of elements $a_{1}, \ldots, a_{t}$ of $I$ is called a filter-regular sequence for $R$ with respect to $I$ if

$$
\left(a_{1}, \ldots, a_{i-1}\right) R:_{R} a_{i} \subseteq\left(a_{1}, \ldots, a_{i-1}\right) R:_{R}\langle I\rangle \text { for } 1 \leq i \leq t .
$$

Assume that $\ell(I)=s$. Then a sequence of elements $a_{1}, \ldots, a_{s}$ of $I$ is called a reduction sequence for $I$ if the initial forms $a_{1}^{*}, \ldots, a_{s}^{*}$ of $a_{1}, \ldots, a_{s}$ in $G$ are of degree one and form a filter-regular sequence for $G$ with respect to $G_{+}$, where $G_{+}$is the ideal generated by all homogeneous elements of positive degree in $G$ and a system of parameters for $F$. If $s=\operatorname{dim} R=d$, a reduction sequence $a_{1}, \ldots, a_{d}$ for $I$ will be called a super-reduction for $I$, if for every relevant highest-dimensional prime ideal $P$ of $G$, the initial forms $a_{1}^{*}, \ldots, a_{d(P)}^{*}$ are a system of parameters for $G /(m G+P)$, where $d(P)=\operatorname{dim} G /(m G+P)$. Suppose $a_{1}, \ldots, a_{d}$ form a super-reduction for $I$ and $J_{d-1}=\left(a_{1}, \ldots, a_{d-1}\right) R:_{R}\langle I\rangle$. Then $j(I)=\lambda_{R}\left(R /\left(J_{d-1}+a_{d} R\right)\right)$, where $\lambda_{R}\left(R /\left(J_{d-1}+a_{d} R\right)\right)$ denotes the length of $R /\left(J_{d-1}+a_{d} R\right)$; see [1]. It is well known that if $R$ has infinite residue field, every ideal has reduction sequences or, if the ideal has maximal analytic spread, super-reductions. For concepts and results about analytic spread, $(j$-)multiplicities and (super-) reduction sequences; see [1, [2], [3, [5, 15, [16] and 21].

Now we will define the $j$-multiplicity for ideals that have possibly non-maximal analytic spread.

Definition 2.1. Let $(R, m, k)$ be either a Noetherian local ring or a standard graded Noetherian algebra over an Artinian local ring $\left(R_{0}, m_{0}\right)$, where $m$ is either the maximal ideal or the homogeneous maximal ideal $\left(m_{0}, R_{1} R\right)$ of $R$ and $k=R / m$ is the residue field. Let $I$ be an $R$-ideal with analytic spread $\ell(I)=s$. Set $G=\operatorname{gr}_{I}(R)$ and $\Gamma_{m}(G)=0:_{G}\langle m\rangle=\bigoplus_{j=0}^{\infty} \Gamma_{m}\left(I^{j} / I^{j+1}\right)$. The module $\Gamma_{m}(G)$ is finitely generated and graded over a standard graded Noetherian algebra over the Artinian local ring $R / m^{t}$ for some integer $t>0$. Furthermore, $\operatorname{dim} \Gamma_{m}(G) \leq s$. So $\Gamma_{m}(G)$ has a Hilbert function that is eventually a polynomial of degree at most $s-1$. Define

$$
j_{s}(I)=(s-1) ! \lim _{i \rightarrow \infty} \frac{\lambda\left(\Gamma_{m}\left(I^{i} / I^{i+1}\right)\right)}{i^{s-1}} .
$$


Notice that $j_{s}(I)=e\left(\Gamma_{m}(G)\right)$ if $\operatorname{dim} \Gamma_{m}(G)=s$ and zero otherwise. When $s=\operatorname{dim} R, j_{s}(I)$ is the usual $j$-multiplicity $j(I)$. In particular if $I$ is $m$-primary, $j_{s}(I)=j(I)=e_{I}(R)$, the Hilbert-Samuel multiplicity.

The following lemma shows that the $j_{s}$-multiplicity does not change modulo the ideal $0::_{R}\langle I\rangle$.

Lemma 2.2. Let $(R, m, k)$ be as in Definition 2.1 with $k$ infinite and $I$ an ideal of $R$. Set $J=0:_{R}\langle I\rangle$ and assume $\bar{R}=R / J \neq 0$. Write $\bar{I}=I \bar{R}$ and $s=\ell(\bar{I})$. Then $s \geq 1$ and the following hold:

(a) $\ell(I)=\ell(\bar{I})=s$;

(b) $j_{s}(I)=j_{s}(\bar{I})$.

Proof. (a) If $s=0$, then $I$ is in the nilradical of $R$ and $\bar{R}=R / J=0$. Hence it is easy to see that $s \geq 1$ and $\ell(I) \geq s$. On the other hand, since $k$ is infinite, there exists an ideal $H \subseteq I$ generated by $s$ elements with $I^{j} \subseteq H I^{j-1}+J$ for $j \gg 0$. So $I^{j} \subseteq H I^{j-1}+J \cap I^{j}$ for sufficiently large $j$. Let $t$ be an integer such that $J=0:_{R} I^{t}$. By the Artin-Rees lemma, there exists $c \geq 0$ so that for all $j \geq c+t$, $I^{j} \cap J=I^{j-c}\left(I^{c} \cap J\right) \subseteq I^{t} J=0$. So $I^{j} \subseteq H I^{j-1}$ for $\bar{j} \gg 0$, i.e., $H$ is a reduction of $I$. Since $H$ is generated by $s$ elements, it follows that $\ell(I) \leq s$.

(b) Let $G=\operatorname{gr}_{I}(R)=\bigoplus_{j=0}^{\infty} I^{j} / I^{j+1}, \bar{G}=\operatorname{gr}_{\bar{I}}(\bar{R})=\bigoplus_{j=0}^{\infty} I^{j} /\left(I^{j} \cap J+I^{j+1}\right)$. By the proof of part (a), $I^{j} \cap J=0$ when $j \gg 0$. Set $\bar{m}=m \bar{R}$, one has $\Gamma_{m}\left(I^{j} / I^{j+1}\right)=\Gamma_{\bar{m}}\left(I^{j} /\left(I^{j} \cap J+I^{j+1}\right)\right)$ for $j \gg 0$. Thus one has $\operatorname{dim} \Gamma_{m}(G)=$ $\operatorname{dim} \Gamma_{\bar{m}}(\bar{G})$. Furthermore $j_{s}(I)=e\left(\Gamma_{m}(G)\right)=e\left(\Gamma_{\bar{m}}(\bar{G})\right)=j_{s}(\bar{I})$ if $\operatorname{dim} \Gamma_{m}(G)=s$, and $j_{s}(I)=0=j_{s}(\bar{I})$ otherwise.

Next we want to prove a result about (super-) reduction sequences. Before doing that, let us recall the bigraded ring $T=\operatorname{gr}_{m}\left(\operatorname{gr}_{I}(R)\right)$ from 2]. Observe $T=\bigoplus_{i, j=0}^{\infty} T_{i j}$, where $T_{i j}=\left(m^{i} I^{j}+I^{j+1}\right) /\left(m^{i+1} I^{j}+I^{j+1}\right)$ and $T_{00}=R / m=k$. First we give a fact about the bigraded ring $T$.

Proposition 2.3. Let $(R, m, k)$ be as in Definition 2.1 and assume that $k$ is infinite. Let $I=\left(a_{1}, \ldots, a_{n}\right) R$ be an ideal with $\ell(I)=s$. Write $x_{i}=\sum_{j=1}^{n} \lambda_{i j} a_{j}$ for $1 \leq i \leq s$ and $\Lambda=\left(\lambda_{i j}\right) \in R^{s n}$. Then there $i s$ a dense open subset $U$ of $k^{s n}$ such that if the image $\bar{\Lambda}=\left(\overline{\lambda_{i j}}\right) \in U$, the images $x_{1}^{o}, \ldots, x_{s}^{o}$ of $x_{1}, \ldots, x_{s}$ in $T_{01}=I / m I$ are a filter-regular sequence for $T$ with respect to the ideal $T_{01} T$ and a system of parameters for $F=G / m G=\bigoplus_{j=0}^{\infty} T_{0 j}$.

Proof. Observe that if $s=0$, the result is obvious. So we may assume $s>0$. First we show there exists a dense open subset $U_{1}$ of $k^{s n}$ such that if the image $\bar{\Lambda} \in U_{1}$, then $x_{1}^{o}, \ldots, x_{s}^{o}$ form a filter-regular sequence with respect to the ideal $T_{01} T$. To do this, let $Z=\left(z_{i j}\right)$ be variables over $R, 1 \leq i \leq s, 1 \leq j \leq n$, $R^{\prime}=R[Z]$ and $T^{\prime}=\operatorname{gr}_{m R^{\prime}}\left(\operatorname{gr}_{I R^{\prime}}\left(R^{\prime}\right)\right)=T[Z]$. Write the generic linear combinations $x_{i}^{\prime}=\sum_{j=1}^{n} z_{i j} a_{j}, 1 \leq i \leq s$. The images $x_{1}^{\prime o}, \ldots, x_{s}^{\prime o}$ in $T_{01}^{\prime}=I R^{\prime} / m I R^{\prime}$ are of degree one (set the degrees of $z_{i j}$ to be zero) and form a filter-regular sequence with respect to $T_{01}^{\prime} T^{\prime}$, i.e., a weakly regular sequence locally at every prime ideal of $\operatorname{Spec}\left(T^{\prime}\right) \backslash V\left(T_{01}^{\prime} T^{\prime}\right)$. So the Koszul complex $K .\left(x_{1}^{\prime o}, \ldots, x_{s}^{\prime o}\right)$ in $T^{\prime}$ is acyclic locally on $\operatorname{Spec}\left(T^{\prime}\right) \backslash V\left(T_{01}^{\prime} T^{\prime}\right)$. Notice $Z-\bar{\Lambda}=\left(z_{i j}-\overline{\lambda_{i j}}\right)$ is an ideal in $k[Z]$ and $K .\left(x_{1}^{\prime o}, \ldots, x_{s}^{o}\right) \otimes_{k[Z]} k[Z] /(Z-\bar{\Lambda})=K .\left(x_{1}^{o}, \ldots, x_{s}^{o}\right)$ is a Koszul complex in $T$. We only need to show that, by avoiding a proper closed subset of $k^{s n}, K .\left(x_{1}^{o}, \ldots, x_{s}^{o}\right)_{Q}$ is acyclic whenever $Q \in \operatorname{Spec}(T) \backslash V\left(T_{01} T\right)$. Observe that $T^{\prime}$ is a finitely generated algebra over the Noetherian domain $k[Z]$ and the zeroth 
Koszul homology $H_{0}\left(x_{1}^{\prime o}, \ldots, x_{s}^{\prime o}\right)=T^{\prime} /\left(x_{1}^{\prime o}, \ldots, x_{s}^{\prime o}\right) T^{\prime}$ is a finite $T^{\prime}$-module. By the Generic Flatness Lemma, there exists an element $0 \neq f \in k[Z]$ such that $T_{f}^{\prime}$ and $H_{0}\left(x_{1}^{\prime o}, \ldots, x_{s}^{\prime o}\right)_{f}$ are all free over $k[Z]_{f}$. Set $U_{1}=k^{s n} \backslash V(f)$. We claim that $U_{1}$ is the desired dense open subset. Indeed, let $\Lambda \in R^{s n}$ with $\bar{\Lambda} \in U_{1}$; then $K .\left(x_{1}^{\prime o}, \ldots, x_{s}^{\prime o}\right)_{f} \otimes_{k[Z]_{f}} k[Z]_{f} /(Z-\bar{\Lambda}) \simeq K .\left(x_{1}^{o}, \ldots, x_{s}^{o}\right)$. For every prime ideal $Q \in \operatorname{Spec}(T) \backslash V\left(T_{01} T\right), T_{Q}^{\prime} /\left(x_{1}^{\prime o}, \ldots, x_{s}^{\prime o}\right) T_{Q}^{\prime}=\left(T_{f}^{\prime} /\left(x_{1}^{\prime o}, \ldots, x_{s}^{\prime o}\right) T_{f}^{\prime}\right)_{\bar{Q}}$ is flat over $k[Z]_{f}$. Thus $\operatorname{Tor}_{i}^{k[Z]_{f}}\left(T_{Q}^{\prime} /\left(x_{1}^{\prime o}, \ldots, x_{s}^{\prime o}\right) T_{Q}^{\prime}, k[Z]_{f} /(Z-\bar{\Lambda})\right)=0$ for $1 \leq i \leq s$, i.e., $K .\left(x_{1}^{o}, \ldots, x_{s}^{o}\right)_{Q}$ is acyclic.

Secondly, by 14 there exists another dense open subset $U_{2}$ of $k^{s n}$ such that if the image $\bar{\Lambda} \in U_{2}$, then $x_{1}, \ldots, x_{s}$ generate a minimal reduction of $I$. Therefore, $x_{1}^{o}, \ldots, x_{s}^{o}$ form a system of parameters for the special fiber $\operatorname{ring} F=G / m G$. Now let $U=U_{1} \cap U_{2}$. Then $U$ is a dense open subset of $k^{s n}$, and it has the required property.

Remark 2.4. In this paper, by abuse of notation we will call $\Lambda=\left(\lambda_{i j}\right) \in R^{s n}$ general elements in $R^{s n}$ as in Proposition 2.3.

As a result of Proposition 2.3, we have the following corollary.

Corollary 2.5. Let $(R, m, k)$ be as in Definition 2.1 and assume that $k$ is infinite. Let $I=\left(a_{1}, \ldots, a_{n}\right) R$ be an ideal with $\ell(I)=s$. Then for general elements $\Lambda=$ $\left(\lambda_{i j}\right) \in R^{s n}, x_{1}, \ldots, x_{s}$ form a reduction sequence for $I$ or, if $s=\operatorname{dim} R$, a superreduction for $I$.

Proof. Notice that if the images $x_{1}^{o}, \ldots, x_{s}^{o}$ in $T_{01}=I / m I$ are a filter-regular sequence with respect to the ideal $T_{01} T$, then the initial forms $x_{1}^{*}, \ldots, x_{s}^{*}$ in the associated graded $\operatorname{ring} G=\operatorname{gr}_{I}(R)$ form a filter-regular sequence of degree one with respect to $G_{+}$; see 2 . Now the first part follows from Proposition 2.3. For the second part, when $s=\operatorname{dim} R=d$, we can just avoid finitely many more proper closed subsets of $k^{d n}$ to assume that $x_{1}, \ldots, x_{d}$ form a super-reduction for the ideal $I$.

Remark 2.6. Corollary 2.5 was first stated by Achilles and Manaresi [1, 2.9], but their proof shows only that one can choose general elements $\left(\lambda_{i j}\right)$ sequentially. Our proof shows that indeed one can choose the general elements all at once.

\section{Formulas for a graded algebra over an Artinian local Ring}

In this section, we will give the multiplicity formula for a standard graded Noetherian algebra $R$ over an Artinian local ring. This formula expresses the multiplicity of $R$ in terms of data associated to prime ideals in $V(I)$, for $I \subseteq R$ any given ideal generated by linear forms.

Theorem 3.1. Let $R=R_{0}\left[R_{1}\right]$ be a standard graded Noetherian ring of dimension $d$ with $\left(R_{0}, m_{0}\right)$ an Artinian local ring. Assume $\left|R_{0} / m_{0}\right|=\infty$. Let $I=\left(a_{1}, \ldots, a_{n}\right) R$ be an ideal generated by homogeneous elements $a_{1}, \ldots, a_{n}$ of degree one. Write ht $I=g$ and $\ell(I)=s$. For general elements $\Lambda=\left(\lambda_{i j}\right) \in R^{s n}$, let $x_{i}=\sum_{j=1}^{n} \lambda_{i j} a_{j}, J_{i-1}=\left(x_{1}, \ldots, x_{i-1}\right) R:_{R}\langle I\rangle, \mathfrak{Q}_{0}=\{q \in \operatorname{Min}(I) \mid \operatorname{dim} R / q=$ $d\}$ and $\mathfrak{Q}_{i}=\left\{q \in \operatorname{Min}\left(J_{i-1}+I\right) \mid \operatorname{dim} R / q=d-i\right\}$, for $1 \leq i \leq s$. Then 
$\ell\left(I_{q} /\left(x_{1}, \ldots, x_{i-1}\right) R_{q}\right)=1$ for every $q \in \mathfrak{Q}_{i}$ with $1 \leq i \leq s$, and

$$
e(R)=\sum_{q \in \mathfrak{Q}_{0}} e_{I_{q}}\left(R_{q}\right) e(R / q)+\sum_{i=\max \{1, g\}}^{s} \sum_{q \in \mathfrak{Q}_{i}} j_{1}\left(\frac{I_{q}}{\left(x_{1}, \ldots, x_{i-1}\right) R_{q}}\right) e(R / q) .
$$

Proof. By Corollary 2.5, there exist general elements $\Lambda=\left(\lambda_{i j}\right) \in R^{s n}$ such that $x_{1}, \ldots, x_{s}$ form a reduction sequence for $I$. In particular by [1, 2.8], $x_{1}, \ldots, x_{s}$ are a filter-regular sequence for $R$ with respect to the ideal $I$. We show that whenever $x_{1}, \ldots, x_{s}$ form a reduction sequence for $I$, then

$$
e(R)=\sum_{q \in \mathfrak{Q}_{0}} \lambda\left(R_{q}\right) e(R / q)+\sum_{i=1}^{s} \sum_{q \in \mathfrak{Q}_{i}} \lambda\left(\frac{R_{q}}{J_{i-1} R_{q}+x_{i} R_{q}}\right) e(R / q) .
$$

We use induction on $d$ to prove this. First when $d=0$, one has $g=0$ and $\mathfrak{Q}_{0}=\{m\}$, where $m=\left(m_{0}, R_{1} R\right)$ is the homogeneous maximal ideal of $R$. Then $e(R)=$ $\lambda(R)=\lambda\left(R_{m}\right)$ and we are done. Let $d \geq 1$ and $\mathfrak{Q}_{0}^{\prime}=\{q \in \operatorname{Min}(R) \mid \operatorname{dim} R / q=$ $d, I \nsubseteq q\}$. By the associativity formula,

$$
e(R)=\sum_{q \in \mathfrak{Q}_{0}} \lambda\left(R_{q}\right) e(R / q)+\sum_{q \in \mathfrak{Q}_{0}^{\prime}} \lambda\left(R_{q}\right) e(R / q)
$$

We may assume $s>0$ as otherwise $\mathfrak{Q}_{0}^{\prime}=\emptyset$ and the result is obvious. Furthermore, if $\operatorname{dim} R / J_{0}<d$, then $\mathfrak{Q}_{0}^{\prime}=\emptyset$ and $\mathfrak{Q}_{i}=\emptyset$ for $1 \leq i \leq s$. The last assertion follows since $x_{i}$ is a non-zero-divisor on $R / J_{i-1}$ for $1 \leq i \leq s$. Assume $\operatorname{dim} R / J_{0}=d$. Since $x_{1}$ is a linear non-zero-divisor on $R / J_{0}$,

$$
\sum_{q \in \mathfrak{Q}_{0}^{\prime}} \lambda\left(R_{q}\right) e(R / q)=e\left(R / J_{0}\right)=e\left(R /\left(J_{0}+x_{1} R\right)\right)
$$

Let $\mathfrak{Q}_{1}^{\prime}=\left\{q \in \operatorname{Min}\left(J_{0}+x_{1} R\right) \mid \operatorname{dim} R / q=d-1, I \nsubseteq q\right\}$, then

$$
\begin{aligned}
e(R)= & \sum_{q \in \mathfrak{Q}_{0}} \lambda\left(R_{q}\right) e(R / q)+e\left(R /\left(J_{0}+x_{1} R\right)\right) \\
= & \sum_{q \in \mathfrak{Q}_{0}} \lambda\left(R_{q}\right) e(R / q)+\sum_{q \in \mathfrak{Q}_{1}} \lambda\left(\frac{R_{q}}{J_{0} R_{q}+x_{1} R_{q}}\right) e(R / q) \\
& +\sum_{q \in \mathfrak{Q}_{1}^{\prime}} \lambda\left(\frac{R_{q}}{J_{0} R_{q}+x_{1} R_{q}}\right) e(R / q) .
\end{aligned}
$$

Similarly, we may suppose $s>1$ as otherwise $\mathfrak{Q}_{1}^{\prime}=\emptyset$ and the result is obvious. Set $\bar{R}=R /\left(J_{0}+x_{1} R\right)$; then $\operatorname{dim} \bar{R}=d-1$. Let $G_{I}\left(J_{0}+x_{1} R, R\right)$ be the initial ideal of $J_{0}+x_{1} R$ in the associated graded ring $G=\operatorname{gr}_{I}(R)$. Then $\bar{G}=\operatorname{gr}_{I}(\bar{R})=$ $G / G_{I}\left(J_{0}+x_{1} R, R\right)$ and $\bar{F}=\bar{G} / \bar{m} \bar{G}=G /\left(G_{I}\left(J_{0}+x_{1} R, R\right)+m G\right)$. We will show $\ell(I \bar{R})=\operatorname{dim} \bar{F}=s-1$. Observe that $x_{1}^{*}, \ldots, x_{s}^{*}$ form a system of parameters for $F=G / m G$. Thus $\operatorname{dim} G /\left(x_{1}^{*} G+m G\right)=s-1$. Since $x_{1} R \subseteq J_{0}+x_{1} R \subseteq J_{1}$, $G_{I}\left(J_{0}+x_{1} R, R\right):_{G}\left\langle G_{+}\right\rangle=G_{I}\left(J_{1}, R\right):_{G}\left\langle G_{+}\right\rangle=x_{1}^{*} G:_{G}\left\langle G_{+}\right\rangle$(see [2, 3.2]). Therefore, $G_{I}\left(J_{0}+x_{1} R, R\right)$ and $x_{1}^{*} G$ have the same relevant associated prime ideals. As $\operatorname{dim} G /\left(x_{1}^{*} G+m G\right)=s-1>0$, it follows that $\operatorname{dim} \bar{F}=$ $\operatorname{dim} G /\left(G_{I}\left(J_{0}+x_{1} R, R\right)+m G\right)=\operatorname{dim} G /\left(x_{1}^{*} G+m G\right)=s-1$.

Let $\overline{x_{2}}, \ldots, \overline{x_{s}}$ be the images of $x_{2}, \ldots, x_{s}$ in $\bar{R}$. From the above argument, $G_{I}\left(J_{0}+x_{1} R, R\right):_{G}\left\langle G_{+}\right\rangle=x_{1}^{*} G:_{G}\left\langle G_{+}\right\rangle$. Hence the initial forms $\overline{x_{2}}{ }^{*}, \ldots,{\overline{x_{s}}}^{*}$ of $\overline{x_{2}}, \ldots, \overline{x_{s}}$ in $\bar{G}$ form a filter-regular sequence for $\bar{G}$ with respect to $\bar{G}_{+}$. As $x_{1}^{*}$ 
is part of a system of parameters of $G / m G, \overline{x_{2}}, \ldots, \overline{x_{s}}$ form a reduction sequence for $\bar{I}$. Observe $\left(J_{0}, x_{1}, \ldots, x_{i-1}\right) R:_{R}\langle I\rangle=J_{i-1}$ for $1 \leq i \leq s$. By the induction hypothesis on $\bar{R}$, we get

$$
e\left(R /\left(J_{0}+x_{1} R\right)\right)=\sum_{i=1}^{s} \sum_{q \in \mathfrak{Q}_{i}} \lambda\left(\frac{R_{q}}{J_{i-1} R_{q}+x_{i} R_{q}}\right) e(R / q) .
$$

Substituting into equation (4) we are done.

Now let $q \in \bigcup_{i=0}^{s} \mathfrak{Q}_{i}$. If $q \in \mathfrak{Q}_{0}$, then $\lambda\left(R_{q}\right)=e_{I_{q}}\left(R_{q}\right)$. So assume $q \in \mathfrak{Q}_{i}$ for some $i \geq 1$. We will show $\ell\left(I_{q} /\left(x_{1}, \ldots, x_{i-1}\right) R_{q}\right)=1$ and

$$
\lambda\left(R_{q} /\left(J_{i-1} R_{q}+x_{i} R_{q}\right)\right)=j_{1}\left(I_{q} /\left(x_{1}, \ldots, x_{i-1}\right) R_{q}\right) .
$$

First we want to see

$$
\lambda\left(R_{q} /\left(J_{i-1} R_{q}+x_{i} R_{q}\right)\right)=j_{1}\left(I\left(R_{q} / J_{i-1} R_{q}\right)\right) .
$$

Let $\bar{R}=R / J_{i-1}$. Since $q \in V\left(J_{i-1}+I\right)$ with $\operatorname{dim} R / q=d-i$, ht $J_{i-1} \geq i-1$ and $x_{i} \in I$ is a non-zero-divisor on $R / J_{i-1}$, one has $\operatorname{dim} \bar{R}=d-i+1$. In the local ring $\bar{R}_{\bar{q}}, 1 \geq \operatorname{dim} \bar{R}_{\bar{q}} \geq$ grade $I \bar{R}_{\bar{q}} \geq 1$. Therefore, $I \bar{R}_{\bar{q}}$ is $q \bar{R}_{\bar{q}}$-primary with $\ell\left(I \bar{R}_{\bar{q}}\right)=\operatorname{dim} \bar{R}_{\bar{q}}=1$. By [1, 3.8 and 2.6], we only need to show the initial form $\bar{x}_{i}{ }^{*}$ of $\overline{x_{i}}$ in the associated graded ring $\operatorname{gr}_{I \bar{R}_{\bar{q}}}\left(\bar{R}_{\bar{q}}\right)$ is filter-regular with respect to $\left(\operatorname{gr}_{I} \bar{R}_{\bar{q}}\left(\bar{R}_{\bar{q}}\right)\right)_{+}$. But this comes from [2,3.2] and the fact that the filter-regular property is preserved under localization.

Next we need to show $\ell\left(I_{q} /\left(x_{1}, \ldots, x_{i-1}\right) R_{q}\right)=1$, and

$$
j_{1}\left(I \bar{R}_{\bar{q}}\right)=j_{1}\left(\frac{I_{q}}{\left(x_{1}, \ldots, x_{i-1}\right) R_{q}}\right) .
$$

For this we only need to apply Lemma 2.2 to the local ring $R_{q} /\left(x_{1}, \ldots, x_{i-1}\right) R_{q}$ and its ideal $I_{q} /\left(x_{1}, \ldots, x_{i-1}\right) R_{q}$.

Finally since $\mathfrak{Q}_{i}=\emptyset, 0 \leq i \leq g-1$, we get equation (3).

Remark 3.2. Theorem 3.1 is motivated by the intersection algorithms constructed by Achilles and Manaresi [2. Indeed the idea behind the original Stückrad-Vogel algorithm of refined intersection theory (cf. [3, Section 3.2], for example), from which [2] evolved, is that the $j$-multiplicity can be calculated by adding together the contributions from top-dimensional components, then cutting by a hyperplane in general position and repeating the process, and so on. Returning to [2, recall that the degrees of cycles $v_{i}(\underline{x}, R)$ of $R$ supported on $V(I)$ are defined by

$$
\begin{gathered}
\operatorname{deg}\left(v_{0}(\underline{x}, R)\right)=\sum_{q \in \mathfrak{Q}_{0}} \lambda\left(R_{q}\right) e(R / q), \\
\operatorname{deg}\left(v_{i}(\underline{x}, R)\right)=\sum_{q \in \mathfrak{Q}_{i}} \lambda\left(\frac{R_{q}}{J_{i-1} R_{q}+x_{i} R_{q}}\right) e(R / q), 1 \leq i \leq s .
\end{gathered}
$$

They defined the multiplicity sequence $c_{0}(I), \ldots, c_{d}(I)$ with respect to the ideal $I$ using the bigraded $\operatorname{ring} T=\operatorname{gr}_{m}\left(\operatorname{gr}_{I}(R)\right)$. They proved that if the images $x_{1}^{o}, \ldots, x_{s}^{o}$ in $T_{01}=I / \mathrm{mI}$ are a filter-regular sequence for $T$ with respect to the ideal $T_{01} T$ and a system of parameters for $F=G / m G$, then $\operatorname{deg}\left(v_{i}(\underline{x}, R)\right)=c_{d-i}(I)[2,4.1]$. The proof of Theorem 3.1 shows that for general elements $\left(\lambda_{i j}\right)$ in $R^{s n}, c_{d}(I)=$ $\sum_{q \in \mathfrak{Q}_{0}} e_{I_{q}}\left(R_{q}\right) e(R / q), c_{d-i}(I)=\sum_{q \in \mathfrak{Q}_{i}} j_{1}\left(\frac{I_{q}}{\left(x_{1}, \ldots, x_{i-1}\right) R_{q}}\right) e(R / q)$ for $1 \leq i \leq s$, and the others are all zero. 
Now we want to apply Theorem 3.1 to some classes of ideals to obtain better formulas. To do this, first we need to recall some facts about residual intersections from [9]. Let $R$ be a Noetherian ring and $I$ an ideal of $R$. Set $H=\left(x_{1}, \ldots, x_{t}\right) R:_{R}$ $I$, where $\left(x_{1}, \ldots, x_{t}\right) R \subsetneq I$. If ht $H \geq t \geq$ ht $I$, then $H$ is said to be a $t$-residual intersection of $I$ with respect to $\left(x_{1}, \ldots, x_{t}\right) R$. Furthermore if $I_{q}=\left(x_{1}, \ldots, x_{t}\right) R_{q}$ for all $q \in V(I)$ with ht $q \leq t$, then $H$ is a geometric t-residual intersection of $I$. Notice that the ideal $H$ is a geometric $t$-residual intersection of $I$ if and only if ht $H \geq t$ and $\operatorname{ht}(H+I) \geq t+1$. An ideal $I$ satisfies condition $G_{t+1}$ if $\mu\left(I_{p}\right) \leq$ ht $p$ for all $p \in V(I)$ such that ht $p \leq t$. Here $\mu(M)$ denotes the least number of generators of a module $M$.

Recall that an ideal $I$ has the Artin-Nagata property $A N_{t}^{-}$if for every $i$ with ht $I \leq i \leq t$ and every geometric $i$-residual intersection $H$ of $I, R / H$ is CohenMacaulay [20]. The ideal $I$ is said to be strongly Cohen-Macaulay (SCM) if the Koszul homology modules of any set of generators of $I$ are Cohen-Macaulay modules. Notice that it suffices to check this property for a fixed system of generators of $I$.

The following corollary shows that condition $G_{t+1}$ and the Artin-Nagata property $A N_{t-1}^{-}$determine how many terms we can simplify in equation (3).

Corollary 3.3. Let $R=R_{0}\left[R_{1}\right]$ be a standard graded Cohen-Macaulay ring with $\left(R_{0}, m_{0}\right)$ an Artinian local ring. Assume $\left|R_{0} / m_{0}\right|=\infty$. Let $I=\left(a_{1}, \ldots, a_{n}\right) R$ be an ideal generated by homogeneous elements $a_{1}, \ldots, a_{n}$ of degree one. Write ht $I=g$, $\ell(I)=s$ and assume that the ideal I satisfies condition $G_{t+1}$ and the Artin-Nagata property $A N_{t-1}^{-}$, where $g \leq t \leq s$. For general elements $\Lambda=\left(\lambda_{i j}\right) \in R^{s n}$, define $x_{i}$ and $\mathfrak{Q}_{i}, 1 \leq i \leq s$, as in Theorem 3.1. Let $H_{i}=\left(x_{1}, \ldots, x_{i}\right) R:_{R} I$, where $0 \leq i \leq s$. Then

$$
\begin{aligned}
e(R)=e(R / I) & +\sum_{i=g+1}^{t} e\left(R /\left(H_{i-1}+I\right)\right) \\
& +\sum_{i=t+1}^{s} \sum_{q \in \mathfrak{Q}_{i}} j_{1}\left(\frac{I_{q}}{\left(x_{1}, \ldots, x_{i-1}\right) R_{q}}\right) e(R / q) .
\end{aligned}
$$

Proof. Let $Z=\left(z_{i j}\right), 1 \leq i \leq s, 1 \leq j \leq n$, be variables over $R$, and $R^{\prime}=R[Z]$. Set $x_{i}^{\prime}=\sum_{j=1}^{n} z_{i j} a_{j}$ and $H_{i}^{\prime}=\left(x_{1}^{\prime}, \ldots, x_{i}^{\prime}\right) R^{\prime}:_{R^{\prime}} I R^{\prime}, 0 \leq i \leq s$. Since $I$ satisfies condition $G_{t+1}$, by [9, 3.2] for each $i$ with $g \leq i \leq t, H_{i}^{\prime}$ is a geometric $i$-residual intersection of $I R^{\prime}$, i.e., ht $H_{i}^{\prime} \geq i$ and ht $\left(H_{i}^{\prime}+I R^{\prime}\right) \geq i+1$. Let $k=R /\left(m_{0}, R_{1} R\right)=$ $R_{0} / m_{0}, \Lambda=\left(\lambda_{i j}\right) \in R^{s n}$ and $\bar{\Lambda}$ be the image of $\Lambda$ in $k^{s n}$. Write $\pi\left(H_{i}^{\prime}\right)$ for the ideal in $R$ generated by the image of $H_{i}^{\prime}$ under the evaluation map sending $z_{i j}$ to $\lambda_{i j}$. By [7. 3.1], for all $i$ with $g \leq i \leq t$, there exists a dense open subset $U_{1}$ of $k^{s n}$ such that ht $\left(\pi\left(H_{i}^{\prime}\right)\right) \geq i$ and $\operatorname{ht}\left(\pi\left(H_{i}^{\prime}\right)+I\right) \geq i+1$ whenever $\bar{\Lambda} \in U_{1}$. Let $U_{2}$ be the dense open subset of $k^{s n}$ as in Theorem 3.1; $U=U_{1} \cap U_{2}$ is still a dense open subset of $k^{s n}$. Let $\Lambda \in R^{s n}$ with $\bar{\Lambda} \in U$. Then for $g \leq i \leq t$, since $\pi\left(H_{i}^{\prime}\right) \subseteq H_{i}, H_{i}$ is also a geometric $i$-residual intersection of $I$ and hence $I_{q}=\left(x_{1}, \ldots, x_{i}\right) R_{q}$ for every $q \in \mathfrak{Q}_{i}$. Now consider $\mathfrak{Q}_{i}$, where $g \leq i \leq t$. If $q \in \mathfrak{Q}_{g}$, since $I_{q}=\left(x_{1}, \ldots, x_{g}\right) R_{q}$ is a complete intersection, $e_{I_{q}}\left(R_{q}\right)=\lambda\left(R_{q} / I_{q}\right)$ if $g=0$, and $j_{1}\left(I_{q} /\left(x_{1}, \ldots, x_{g-1}\right) R_{q}\right)=e_{I_{q}}\left(R_{q}\right)=$ $\lambda\left(R_{q} / I_{q}\right)$ if $g>0$. Fix $i$ with $g+1 \leq i \leq t$ and let $J_{i-1}$ be defined as in Theorem 3.1. Since $I$ satisfies $A N_{t-1}^{-}, R / H_{i-1}$ is Cohen-Macaulay and therefore $J_{i-1}=H_{i-1}$. Also from [20, 1.7], ht $\left(J_{i-1}+I\right)=i$ and hence $\mathfrak{Q}_{i} \neq \emptyset$. Let $q \in \mathfrak{Q}_{i}$. By equation (5), $j_{1}\left(I_{q} /\left(x_{1}, \ldots, x_{i-1}\right) R_{q}\right)=\lambda\left(R_{q} /\left(J_{i-1} R_{q}+x_{i} R_{q}\right)\right)=\lambda\left(R_{q} /\left(H_{i-1}+I\right)_{q}\right)$. Applying 
Theorem 3.1 and the associativity formula,

$$
\begin{aligned}
e(R)= & \sum_{q \in \mathfrak{Q}_{g}} \lambda\left(R_{q} / I_{q}\right) e(R / q) \\
& +\sum_{i=g+1}^{t} \sum_{q \in \mathfrak{Q}_{i}} \lambda\left(R_{q} /\left(H_{i-1}+I\right)_{q}\right) e(R / q) \\
& +\sum_{i=t+1}^{s} \sum_{q \in \mathfrak{Q}_{i}} j_{1}\left(\frac{I_{q}}{\left(x_{1}, \ldots, x_{i-1}\right) R_{q}}\right) e(R / q) \\
= & e(R / I)+\sum_{i=g+1}^{t} e\left(R /\left(H_{i-1}+I\right)\right) \\
& +\sum_{i=t+1}^{s} \sum_{q \in \mathfrak{Q}_{i}} j_{1}\left(\frac{I_{q}}{\left(x_{1}, \ldots, x_{i-1}\right) R_{q}}\right) e(R / q) .
\end{aligned}
$$

Remark 3.4. In Corollary 3.3, we can replace the Artin-Nagata property $A N_{t-1}^{-}$ by SCM. Indeed if $I$ is an SCM ideal which satisfies condition $G_{t+1}$, then by 9 , 3.3] (see also [8, 3.1]), for $g \leq i \leq t, H_{i}^{\prime}$ is a geometric $i$-residual intersection of $I R^{\prime}, R^{\prime} / H_{i}^{\prime}$ is Cohen-Macaulay and ht $H_{i}^{\prime}=i$. By the proof of Corollary 3.3, for general elements $\Lambda=\left(\lambda_{i j}\right) \in R^{s n}, H_{i}$ is a geometric $i$-residual intersection of $I$ for $g \leq i \leq t$. Notice $Z-\Lambda=\left(z_{i j}-\lambda_{i j}\right)$ is a regular sequence on both $R^{\prime}$ and $R^{\prime} / I R^{\prime}$. For $g+1 \leq i \leq t$, write $\pi\left(H_{i-1}^{\prime}\right)$ for the image modulo $(Z-\Lambda) R^{\prime}$. By [9, 4.7] (see also [13, 10.4]), we have $\pi\left(H_{i-1}^{\prime}\right)=H_{i-1}$ and $R / H_{i-1}$ is also Cohen-Macaulay. Hence $J_{i-1}=H_{i-1}$. Furthermore by the proof of [20, 1.7], ht $\left(J_{i-1}+I\right)=i$ and therefore $\mathfrak{Q}_{i} \neq \emptyset$. The remaining proof is the same as that of Corollary 3.3.

When the ideal $I$ is a complete intersection for every $q \in V(I)$ with ht $q \leq t$, or is perfect of height 2 satisfying condition $G_{t+1}$, or is Gorenstein of height 3 satisfying condition $G_{t+1}$, we obtain nicer formulas.

Corollary 3.5. Let $R=R_{0}\left[R_{1}\right]$ be a standard graded Cohen-Macaulay ring with $\left(R_{0}, m_{0}\right)$ an Artinian local ring. Assume $\left|R_{0} / m_{0}\right|=\infty$. Let $I=\left(a_{1}, \ldots, a_{n}\right) R$ be an ideal generated by homogeneous elements $a_{1}, \ldots, a_{n}$ of degree one. Write ht $I=g$ and $\ell(I)=s$. For general elements $\Lambda=\left(\lambda_{i j}\right) \in R^{s n}$, let $\Lambda_{i-1}$ be the submatrix consisting of the first $i-1$ rows of $\Lambda$ and define $x_{i}, \mathfrak{Q}_{i}$ as before, for $1 \leq i \leq s$.

(a) Assume $I_{q}$ is a complete intersection for every $q \in V(I)$ with ht $q \leq t$, where $g \leq t \leq s$. Then

$$
e(R)=e(R / I)+\sum_{i=g+1}^{t} e\left(R /\left(F_{i-1}+I\right)\right)+\sum_{i=t+1}^{s} \sum_{q \in \mathfrak{Q}_{i}} j_{1}\left(\frac{I_{q}}{\left(x_{1}, \ldots, x_{i-1}\right) R_{q}}\right) e(R / q),
$$

where $F_{i-1}=\operatorname{Fitt}_{0}\left(I /\left(x_{1}, \ldots, x_{i-1}\right) R\right)$. 
(b) Assume $I$ is a perfect ideal of height 2 which satisfies condition $G_{t+1}$. Write $\mu(I)=n$, and let $X_{n \times(n-1)}=\left(x_{i j}\right)$ be a matrix such that $I=I_{n-1}(X)$. Then

$$
\begin{aligned}
e(R)=e(R / I) & +\sum_{i=3}^{t} e\left(R /\left(I_{n}\left(X \mid \Lambda_{i-1}^{T}\right)+I\right)\right) \\
& +\sum_{i=t+1}^{s} \sum_{q \in \mathfrak{Q}_{i}} j_{1}\left(\frac{I_{q}}{\left(x_{1}, \ldots, x_{i-1}\right) R_{q}}\right) e(R / q) .
\end{aligned}
$$

(c) Assume $I$ is a perfect Gorenstein ideal of height 3 which satisfies condition $G_{t+1}$. Write $\mu(I)=n$, and let $X_{n \times n}=\left(x_{i j}\right)$ be an alternating matrix such that $I=\operatorname{Pf}_{n-1}(X)$ (the ideal generated by the $n-1$ by $n-1$ Pfaffians of $X$ ). Let $T_{i-1}=\left(\begin{array}{cc}X & \Lambda_{i-1}^{T} \\ -\Lambda_{i-1} & 0\end{array}\right)$, define $J\left(T_{i-1}\right)$ to be the $R$-ideal generated by the Pfaffians of all principal submatrices of $T_{i-1}$ which contain $X$ for $4 \leq i \leq t$ (see [13]). Then

$e(R)=e(R / I)+\sum_{i=4}^{t} e\left(R /\left(J\left(T_{i-1}\right)+I\right)\right)+\sum_{i=t+1}^{s} \sum_{q \in \mathfrak{Q}_{i}} j_{1}\left(\frac{I_{q}}{\left(x_{1}, \ldots, x_{i-1}\right) R_{q}}\right) e(R / q)$.

Proof. In each of the three cases, $I$ satisfies condition $G_{t+1}$. Write $k=R /\left(m_{0}, R_{1} R\right)$ $=R_{0} / m_{0}$. By the proof of Corollary 3.3, for all $i$ with $g \leq i \leq t$, there is a dense open subset $U \subseteq k^{s n}$ such that whenever $\bar{\Lambda}=\left(\overline{\lambda_{i j}}\right) \in U, H_{i}=\left(x_{1}, \ldots, x_{i}\right) R:_{R} I$ is a geometric $i$-residual intersection of $I$, and equation (3) holds. Set $\Lambda=\left(\lambda_{i j}\right) \in R^{s n}$ with $\bar{\Lambda} \in U$.

(a) As in the proof of Corollary 3.3, let $Z=\left(z_{i j}\right), 1 \leq i \leq s, 1 \leq j \leq n$, be variables over $R$ and $R^{\prime}=R[Z]$. Let $x_{i}^{\prime}=\sum_{j=1}^{n} z_{i j} a_{j}, H_{i}^{\prime}=\left(x_{1}^{\prime}, \ldots, x_{i}^{\prime}\right) R^{\prime}:_{R^{\prime}} I R^{\prime}$ and $J_{i}=\left(x_{1}, \ldots, x_{i}\right) R:_{R}\langle I\rangle$ for $0 \leq i \leq s$. Consider the local ring $R_{q}$, where $q \in V(I)$ with ht $q=t$. Let $g^{\prime}=$ ht $I_{q}$. We may assume $I_{q}=\left(a_{1}, \ldots, a_{g^{\prime}}\right)_{q}$ after elementary transformations. There is an invertible $n$ by $n$ matrix $E$ over $R_{q}$ such that $\left(a_{1}, \ldots, a_{n}\right)^{T}=E\left(a_{1}, \ldots, a_{g^{\prime}}, 0, \ldots, 0\right)^{T}$. Fix $i$ with $g^{\prime}+1 \leq i \leq t$. Observe that $R_{q}^{\prime}=R_{q}[Z]$ and $\left(H_{i-1}^{\prime}\right)_{q}=\left(x_{1}^{\prime}, \ldots, x_{i-1}^{\prime}\right) R_{q}^{\prime}:_{R_{q}^{\prime}} I R_{q}^{\prime}$. Let $Z_{i-1}$ be the submatrix of $Z=\left(z_{i j}\right)$ generated by the first $i-1$ rows. Then $\varphi=Z_{i-1} E$ is a generic $i-1$ by $n$ matrix over $R_{q}$. If we write $\varphi=(V \mid W)$, where $V$ is a $i-1$ by $g^{\prime}$ submatrix, then $\left(x_{1}^{\prime}, \ldots, x_{i-1}^{\prime}\right)^{T}=V\left(a_{1}, \ldots, a_{g^{\prime}}\right)^{T}$. By [9, 3.4], $\left(H_{i-1}^{\prime}\right)_{q}=$ $\left(x_{1}^{\prime}, \ldots, x_{i-1}^{\prime}\right)_{q}+I_{g^{\prime}}(V)_{q}$.

In the Cohen-Macaulay local ring $R_{q}^{\prime}$, since $I R_{q}^{\prime}$ is a complete intersection, the ideal $I R_{q}^{\prime}$ is strongly Cohen-Macaulay and satisfies condition $G_{t+1}$. By the same argument of Remark 3.4, we have $R_{q} /\left(H_{i-1}\right)_{q}$ is Cohen-Macaulay and $\left(J_{i-1}\right)_{q}=$ $\left(H_{i-1}\right)_{q}=\pi\left(\left(H_{i-1}^{\prime}\right)_{q}\right)=\left(x_{1}, \ldots, x_{i-1}\right)_{q}+I_{g^{\prime}}(\pi(V))_{q}$.

Observe there is an exact sequence

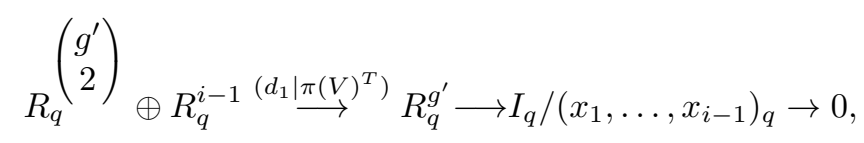

where $d_{1}$ is the first derivation in the Koszul complex $K\left(a_{1}, \ldots, a_{g^{\prime}}\right)$ :

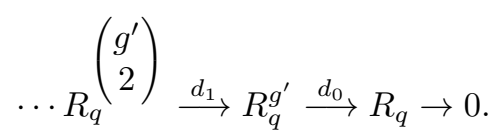


So we have

$$
\operatorname{Fitt}_{0}\left(I /\left(x_{1}, \ldots, x_{i-1}\right)\right)_{q}+I_{q}=\left(H_{i-1}\right)_{q}+I_{q}=I_{g^{\prime}}(\pi(V))_{q}+I_{q} .
$$

Thus $\left(J_{i-1}+I\right)_{q}=\left(H_{i-1}+I\right)_{q}=\operatorname{Fitt}_{0}\left(I /\left(x_{1}, \ldots, x_{i-1}\right)\right)_{q}+I_{q}$.

Finally, let $q \in V(I)$ with ht $q=t$ and ht $I_{q}=g$. For every $i$ with $g+1 \leq i \leq t$, $R_{q} /\left(H_{i-1}\right)_{q}$ is Cohen-Macaulay and hence ht $\left(J_{i-1}+I\right)_{q}=i$ (see the proof of [20, 1.7]). Therefore $\mathfrak{Q}_{i} \neq \emptyset$ for every $i$ with $g+1 \leq i \leq t$. We are done by the proof of Corollary 3.3 and the associativity formula.

(b) Let $R^{\prime}=R[W, Z]$, where $W=\left(w_{i j}\right)$ is a generic $n \times(n-1)$ matrix, $Z=\left(z_{i j}\right)$ is a generic $s \times n$ matrix and $I^{\prime}=I_{n-1}(W)=\left(\Delta_{1}, \ldots, \Delta_{n}\right)$, in an obvious notation. Set $\left(x_{1}^{\prime}, \ldots, x_{s}^{\prime}\right)^{T}=Z\left(\Delta_{1}, \ldots, \Delta_{n}\right)^{T}$ and $H_{i}^{\prime}=\left(x_{1}^{\prime}, \ldots, x_{i}^{\prime}\right) R^{\prime}:_{R^{\prime}} I^{\prime} R^{\prime}$ for $0 \leq i \leq s$. Fix $3 \leq i \leq t$. By [8, 4.1], we have $H_{i-1}^{\prime}=I_{n}\left(W \mid Z_{i-1}^{T}\right)$. Let $(W-X, Z-\Lambda)=$ $\left(w_{i j}-x_{i j} ; z_{i j}-\lambda_{i j}\right)$ and write $\pi\left(H_{i-1}^{\prime}\right)$ for the image modulo $(W-X, Z-\Lambda) R^{\prime}$. By a similar argument as in the proof of $[13,10.5]$, we have that $R / H_{i-1}$ is CohenMacaulay and $H_{i-1}=\pi\left(H_{i-1}^{\prime}\right)=I_{n}\left(X \mid \Lambda_{i-1}^{T}\right)$. Hence $J_{i-1}=H_{i-1}=I_{n}\left(X \mid \Lambda_{i-1}^{T}\right)$, and we are done by the proof of Corollary 3.3.

(c) By the proof of [13, 10.5], for each $i$ with $4 \leq i \leq t$, we have that $R / H_{i-1}$ is Cohen-Macaulay and $H_{i-1}=J\left(T_{i-1}\right)$. Hence $J_{i-1}=H_{i-1}=J\left(T_{i-1}\right)$, and we are done by the proof of Corollary 3.3.

\section{Homogeneous inClusions of GRADED ALGEBRAS OVER AN ARTINIAN LOCAL RING}

In this section, we will consider homogeneous inclusions of two standard graded Noetherian algebras over an Artinian local ring. First we consider the case where these two rings have the same dimension.

Theorem 4.1. Let $A=A_{0}\left[A_{1}\right] \subseteq B=B_{0}\left[B_{1}\right]$ be a homogeneous inclusion of standard graded Noetherian rings of the same dimension d. Let $A_{0}=B_{0}=$ $R_{0}$ be an Artinian local ring with maximal ideal $m_{0}$ and $\left|R_{0} / m_{0}\right|=\infty$. Write $A_{1} A=\left(a_{1}, \ldots, a_{n}\right) A$, where $a_{1}, \ldots, a_{n}$ are homogeneous elements of degree one, and ht $A_{1} B=g$. Let $\mathfrak{P}=\{p \in \operatorname{Spec}(A) \mid \operatorname{dim} A / p=\operatorname{dim} A\}$ and assume that $A_{p}$ is reduced and $\operatorname{rank}_{A_{p}} B_{p}=r$ for every prime ideal $p \in \mathfrak{P}$. For general elements $\Lambda=\left(\lambda_{i j}\right) \in R_{0}^{d n}$, define $x_{i}, J_{i}, \mathfrak{Q}_{i}, 0 \leq i \leq d$, as before. Then

$$
\begin{aligned}
e(B)=r e(A) & +\sum_{q \in \mathfrak{Q}_{0}} e_{A_{1} B_{q}}\left(B_{q}\right) e(B / q) \\
& +\sum_{i=\max \{1, g\}}^{d-1} \sum_{q \in \mathfrak{Q}_{i}} j_{1}\left(\frac{A_{1} B_{q}}{\left(x_{1}, \ldots, x_{i-1}\right) B_{q}}\right) e(B / q) .
\end{aligned}
$$

Proof. Recall that $G=\operatorname{gr}_{A_{1} B}(B)$ and $T=\operatorname{gr}_{m}\left(\operatorname{gr}_{A_{1} B}(B)\right)$, where $m=\left(m_{0}, B_{1} B\right)$ is the homogeneous maximal ideal of $B$. Then $F=G / m G \simeq A / m_{0} A$ (see [17]). Since $\operatorname{dim} A / m_{0} A=\operatorname{dim} A=d, \ell\left(A_{1} B\right)=d$. By Proposition 2.3, for general elements $\Lambda=\left(\lambda_{i j}\right) \in R_{0}^{d n}$, the images $x_{1}^{o}, \ldots, x_{d}^{o}$ of $x_{1}, \ldots, x_{d}$ in $T_{01}=I / m I$ are a filter-regular sequence with respect to the ideal $T_{01} T$ and a system of parameters for $F$. By the proof of Theorem 3.1, equation (3) holds for such $\Lambda$. Thus we only need to show

$$
r e(A)=j_{1}\left(A_{1} B_{m} /\left(x_{1}, \ldots, x_{d-1}\right) B_{m}\right) .
$$


By Remark 3.2 and [2, Theorem 4.1],

$$
j_{1}\left(A_{1} B_{m} /\left(x_{1}, \ldots, x_{d-1}\right) B_{m}\right)=c_{0}\left(A_{1} B\right)=j_{d}\left(A_{1} B\right) .
$$

By [17, 6.1], we know $e(G)=e(B)=r e(A)+e_{\infty}(A, B)$, where $e_{\infty}(A, B)=$ $e\left(G / 0:_{G}\left\langle B_{1} G\right\rangle\right)$. Since $B_{1} B$ and $m$ have the same radical, $0:_{G}\left\langle B_{1} G\right\rangle=$ $0:_{G}\langle m G\rangle$. Therefore $j_{d}\left(A_{1} B\right)=e\left(0:_{G}\langle m G\rangle\right)=e(G)-e_{\infty}(A, B)=\operatorname{re}(A)$ (see also [21]).

Theorem 4.1 is a generalization of Theorem 1.1. When $B$ is equidimensional, we have the following corollary.

Corollary 4.2. In the same setting as Theorem 4.1, assume $B$ is equidimensional and let $\mathfrak{Q}^{\prime}=\left\{q \in V\left(A_{1} B\right) \cap \operatorname{Proj}(B) \mid \ell\left(A_{1} B_{q}\right)=\operatorname{dim} B_{q}\right\}$ as in Proposition 1.2. For general elements $\Lambda=\left(\lambda_{i j}\right) \in R_{0}^{d n}$, define $x_{i}, J_{i}$ and $\mathfrak{Q}_{i}, 0 \leq i \leq d$, as before. Then

$$
\begin{aligned}
e(B)=r e(A) & +\sum_{q \in \mathfrak{Q}^{\prime}} j\left(A_{1} B_{q}\right) e(B / q) \\
& +\sum_{i=\max \{1, g\}} \sum_{q \in \mathfrak{Q}_{i} \backslash \mathfrak{Q}^{\prime}} j_{1}\left(\frac{A_{1} B_{q}}{\left(x_{1}, \ldots, x_{i-1}\right) B_{q}}\right) e(B / q) .
\end{aligned}
$$

Proof. Let $q \in \mathfrak{Q}^{\prime}$. First we show that $q$ is contained in $\mathfrak{Q}_{i}$ for some $i$ with $g \leq i \leq$ $d-1$. Indeed for such $q$, write $\ell\left(A_{1} B_{q}\right)=\operatorname{dim} B_{q}=i$. Then $J_{i-1}+A_{1} B \subseteq q$, since otherwise $\left(J_{i-1}\right)_{q}=B_{q}$ and $\sqrt{A_{1} B_{q}}=\sqrt{\left(x_{1}, \ldots, x_{i-1}\right) B_{q}}$, which contradicts the equality $\ell\left(A_{1} B_{q}\right)=i$. Also $\operatorname{dim} B / q=d-i$ because $B$ is equidimensional. Thus $q \in \mathfrak{Q}_{i}$. Notice that $\mathfrak{Q}^{\prime}$ is a fixed finite set which does not depend on $x_{1}, \ldots, x_{d}$. Moreover $R_{0} / m_{0}$ is a subfield of $k(q)=B_{q} / q B_{q}$ for every prime ideal $q$ of $B$. By avoiding finitely many more proper closed subsets of $k^{d n}$, we can choose general elements $\Lambda=\left(\lambda_{i j}\right) \in R_{0}^{d n}$ such that $x_{1}, \ldots, x_{i}$ form a super-reduction for the ideal $A_{1} B_{q}$ for each $q \in \mathfrak{Q}^{\prime}$ with ht $q=i$. Thus

$$
j_{1}\left(\frac{A_{1} B_{q}}{\left(x_{1}, \ldots, x_{i-1}\right) B_{q}}\right)=\lambda\left(B_{q} /\left(J_{i-1} B_{q}+x_{i} B_{q}\right)\right)=j\left(A_{1} B_{q}\right)
$$

[1]. We are done by applying Theorem 4.1.

From Corollary 4.2, we can see that we have indeed added extra terms to the right-hand side of inequality (2) in Proposition 1.2, to obtain equality.

When $B$ is a domain with the same dimension as that of $A$, we have the following corollary.

Corollary 4.3. Let $A=k\left[A_{1}\right] \subseteq B=k\left[B_{1}\right]$ be a homogeneous inclusion of standard graded Noetherian domains of the same dimension $d$, where $k$ is an infinite field. Write $A_{1} A=\left(a_{1}, \ldots, a_{n}\right) A$, where $a_{1}, \ldots, a_{n}$ are homogeneous elements of degree one, and ht $A_{1} B=g$. Let $r=[L: K]$, where $L=\operatorname{Quot}(B)$ and $K=\operatorname{Quot}(A)$. For general elements $\Lambda=\left(\lambda_{i j}\right) \in k^{d n}$, define $x_{i}, J_{i-1}$, and $\mathfrak{Q}_{i}$, $1 \leq i \leq d$, as before. Then

$$
e(B)=r e(A)+\sum_{i=g}^{d-1} \sum_{q \in \mathfrak{Q}_{i}} j_{1}\left(\frac{A_{1} B_{q}}{\left(x_{1}, \ldots, x_{i-1}\right) B_{q}}\right) e(B / q) .
$$

By Theorem 4.1, and Corollaries 3.3 and 3.5, it is easy to get the following corollary. 
Corollary 4.4. In the same setting as Theorem 4.1, assume B is Cohen-Macaulay. For general elements $\Lambda=\left(\lambda_{i j}\right) \in R_{0}^{d n}$, define $x_{i}, H_{i-1}$ and $\Lambda_{i-1}, 1 \leq i \leq d$, as before.

(a) Assume the ideal $A_{1} B$ satisfies condition $G_{d}$ and the Artin-Nagata property $A N_{d-2}^{-}$. Then

$$
e(B)=r e(A)+e\left(B / A_{1} B\right)+\sum_{i=g+1}^{d-1} e\left(B /\left(H_{i-1}+A_{1} B\right)\right) .
$$

(b) Assume $A_{1} B_{q}$ is a complete intersection for every $q \in \operatorname{Proj}(B)$. Then

$$
e(B)=r e(A)+e\left(B / A_{1} B\right)+\sum_{i=g+1}^{d-1} e\left(B /\left(F_{i-1}+A_{1} B\right)\right),
$$

where $F_{i-1}=\operatorname{Fitt}_{0}\left(A_{1} B /\left(x_{1}, \ldots, x_{i-1}\right) B\right)$.

(c) Assume $A_{1} B$ is a perfect ideal of height 2 which satisfies condition $G_{d}$. Write $\mu\left(A_{1} B\right)=n$ and let $X_{n \times(n-1)}$ be a matrix such that $A_{1} B=I_{n-1}(X)$. Then

$$
e(B)=r e(A)+e\left(B / A_{1} B\right)+\sum_{i=3}^{d-1} e\left(B /\left(I_{n}\left(X \mid \Lambda_{i-1}^{T}\right)+A_{1} B\right)\right) .
$$

(d) Assume $A_{1} B$ is a perfect Gorenstein ideal of height 3 which satisfies condition $G_{d}$. Write $\mu\left(A_{1} B\right)=n$ and let $X_{n \times n}$ be an alternating matrix such that $A_{1} B=$ $\mathrm{Pf}_{n-1}(X)$. Let $T_{i-1}=\left(\begin{array}{cc}X & \Lambda_{i-1}^{T} \\ -\Lambda_{i-1} & 0\end{array}\right)$ and $J\left(T_{i-1}\right)$ be the B-ideal generated by the Pfaffians of all principal submatrices of $T_{i-1}$ which contain $X$ for $4 \leq i \leq d-1$. Then

$$
e(B)=r e(A)+e\left(B / A_{1} B\right)+\sum_{i=4}^{d-1} e\left(B /\left(J\left(T_{i-1}\right)+A_{1} B\right)\right) .
$$

Remark 4.5. In part (a) of Corollary 4.4, we can replace the Artin-Nagata property $A N_{d-2}^{-}$by the SCM (see the argument of Remark 3.4).

Now we consider the case when $\operatorname{dim} A \neq \operatorname{dim} B$. Let $k$ be an infinite field and $R$ a finitely generated $k$-algebra. Recall that $\operatorname{deg}_{k}(R)=\min \left\{\operatorname{rank}_{S} R\right\}$, where $S$ ranges over all Noether normalizations of $R$. If $R=k\left[a_{1}, \ldots, a_{n}\right]$, where $a_{1}, \ldots, a_{n}$ are homogeneous elements of degree one, $\operatorname{deg}_{k}(R)=e(R)=\operatorname{rank}_{S} R$, for any Noether normalization $S$ of $R$ which is a $k$-algebra generated by linear combinations of $a_{1}, \ldots, a_{n}$ [17].

Write $\operatorname{dim} R=d$. Let $Y=\left(y_{i j}\right), 1 \leq i \leq d, 1 \leq j \leq n$, be variables over $R$. Consider the generic linear combinations $y_{i}=\sum_{j=1}^{n} y_{i j} a_{j}, 1 \leq i \leq d$, regarded as elements of $R^{\prime}=R \otimes_{k} k^{\prime}=k^{\prime}\left[a_{1}, \ldots, a_{n}\right]$, where $k^{\prime}=k\left(y_{i j}\right)$. By [6, 7.3], $S^{\prime}=k^{\prime}\left[y_{1}, \ldots, y_{d}\right] \subseteq R^{\prime}$ is a finite homogeneous inclusion. Hence $\operatorname{deg}_{k^{\prime}}\left(R^{\prime}\right)=$ $\operatorname{rank}_{S^{\prime}} R^{\prime}=e\left(R^{\prime}\right)=e(R)=\operatorname{deg}_{k}(R)$.

For $A=A_{0}\left[A_{1}\right] \subseteq B=B_{0}\left[B_{1}\right]$, a homogeneous inclusion of standard graded Noetherian rings with $A_{0}=B_{0}=R_{0}$ Artinian local, the associated graded ring $G=\operatorname{gr}_{A_{1} B}(B)=R_{0}\left[A_{1} T\right]\left[\overline{B_{1}}\right]$, where $T$ is a variable over $B$ and $\overline{B_{1}}$ is the image of $B_{1}$ in $G$. By assigning bidegree $(1,0)$ to the elements of $\overline{B_{1}}$ and bidegree $(0,1)$ to the elements of $A_{1} T, G=\bigoplus_{i, j=0}^{\infty} G_{i j}$ is a bigraded ring with $\bigoplus_{j=0}^{\infty} G_{0 j}=R_{0}\left[A_{1} T\right] \simeq A$ (see [17]). Thus we can think that $A \subseteq G$ and $A_{p} \otimes_{A} G$ is a standard graded Noetherian $A_{p}$-algebra for every prime ideal $p$ of $A$. 
Proposition 4.6. Let $A=A_{0}\left[A_{1}\right] \subseteq B=B_{0}\left[B_{1}\right]$ be a homogeneous inclusion of standard graded Noetherian rings with $\operatorname{dim} A=s$ and $\operatorname{dim} B=d$. Let $A_{0}=$ $B_{0}=R_{0}$ be an Artinian local ring with maximal ideal $m_{0}$ and $\left|R_{0} / m_{0}\right|=\infty$. Write $A_{1} A=\left(a_{1}, \ldots, a_{n}\right) A$, where $a_{1}, \ldots, a_{n}$ are homogeneous elements of degree one. Let $\mathfrak{P}=\{p \in \operatorname{Spec}(A) \mid \operatorname{dim} A / p=\operatorname{dim} A\}$ and assume that $A_{p}$ is reduced, $\operatorname{dim} A_{p} \otimes_{A} G=d-s$ and $\operatorname{deg}_{A_{p}}\left(A_{p} \otimes_{A} G\right)=r$ for every prime ideal $p \in \mathfrak{P}$, where $G=\operatorname{gr}_{A_{1} B}(B)$. For general elements $\Lambda=\left(\lambda_{i j}\right) \in R_{0}^{s n}$, let $x_{i}=\sum_{j=1}^{n} \lambda_{i j} a_{j}$, $1 \leq i \leq s, J_{s-1}=\left(x_{1}, \ldots, x_{s-1}\right) B:_{B}\left\langle A_{1} B\right\rangle$ and let $\mathfrak{Q}_{s}$ be the set of all prime ideals $q$ in $\operatorname{Min}\left(J_{s-1}+A_{1} B\right)$ with $\operatorname{dim} B / q=d-s$. Then

$$
\sum_{q \in \mathfrak{Q}_{s}} j_{1}\left(\frac{A_{1} B_{q}}{\left(x_{1}, \ldots, x_{s-1}\right) B_{q}}\right) e(B / q)=r e(A) .
$$

Proof. Observe $\ell\left(A_{1} B\right)=\operatorname{dim} A=s$ (see the proof of Theorem 4.1). By Proposition 2.3 and 2] (see Remark 3.2), for general elements $\Lambda=\left(\lambda_{i j}\right) \in R_{0}^{s n}$,

$$
\sum_{q \in \mathfrak{Q}_{s}} j_{1}\left(\frac{A_{1} B_{q}}{\left(x_{1}, \ldots, x_{s-1}\right) B_{q}}\right) e(B / q)=\operatorname{deg} v_{s}(\underline{x}, B)=\operatorname{deg} v_{s}\left(\underline{x}^{*}, G\right)=c_{d-s}\left(A_{1} B\right),
$$

which does not depend on the choice of $\Lambda$. We only need to show $c_{d-s}\left(A_{1} B\right)=$ $r e(A)$. Observe $G=R_{0}\left[A_{1} T\right]\left[\overline{B_{1}}\right]$ and $\left(A_{1} T\right) G=G_{+}=\bigoplus_{i=0}^{\infty} \bigoplus_{j=1}^{\infty} G_{i j}$. When $s=d$, by the proof of Theorem 4.1, $c_{0}\left(A_{1} B\right)=\operatorname{deg} v_{d}\left(\underline{x}^{*}, G\right)=j\left(\left(A_{1} T\right) G\right)=$ $\left(\operatorname{rank}_{A} G\right) \cdot e(A)=\operatorname{re}(A)$. Suppose $s<d$ and let $l=d-s$. Set $A=R_{0}\left[A_{1}\right]=$ $R_{0}\left[a_{1}, \ldots, a_{n}\right] \subseteq B=R_{0}\left[B_{1}\right]=R_{0}\left[b_{1}, \ldots, b_{\tau}\right]$. Then $A \simeq R_{0}\left[a_{1} T, \ldots, a_{n} T\right] \subseteq$ $G=R_{0}\left[a_{1} T, \ldots, a_{n} T, \overline{b_{1}}, \ldots, \overline{b_{\tau}}\right]$, where $\overline{b_{1}}, \ldots, \overline{b_{\tau}}$ are the images of $b_{1}, \ldots, b_{\tau}$ in $G_{10}$. We may assume $A=R_{0}\left[a_{1} T, \ldots, a_{n} T\right]=R_{0}\left[G_{01}\right]$. Let $\widetilde{R_{0}}=R_{0}[Z, Y, W]$ and $R_{0}^{\prime}=\left(\widetilde{R_{0}}\right)_{m_{0} \widetilde{R_{0}}}$, where $Z=\left(z_{i j}\right), 1 \leq i \leq s, 1 \leq j \leq n, Y=\left(y_{\mu \nu}\right), 1 \leq$ $\mu \leq l, 1 \leq \nu \leq \tau, W=\left(w_{\alpha \beta}\right), 1 \leq \alpha \leq l, 1 \leq \beta \leq n+l$, are variables over $G$. Let $G^{\prime}=G \otimes_{R_{0}} R_{0}^{\prime}=R_{0}^{\prime}\left[G_{10}, G_{01}\right], A^{\prime}=A \otimes_{R_{0}} R_{0}^{\prime}=R_{0}^{\prime}\left[G_{01}\right]$ and $A^{\prime \prime}=$ $R_{0}^{\prime}\left[G_{01}, y_{1}^{\prime}, \ldots, y_{l}^{\prime}\right]$, where $y_{\mu}^{\prime}=\sum_{\nu=1}^{\tau} y_{\mu \nu} \overline{b_{\nu}}$ for $1 \leq \mu \leq l$. For every $p^{\prime} \in \operatorname{Spec}\left(A^{\prime}\right)$ with $\operatorname{dim} A^{\prime} / p^{\prime}=\operatorname{dim} A^{\prime}=s, p^{\prime}=p A^{\prime}$ for some $p \in \mathfrak{P}$. Hence $A_{p^{\prime}}^{\prime}$ is a field, $A_{p^{\prime}}^{\prime} \otimes_{A^{\prime}} G^{\prime}$ is a finitely generated standard graded $A_{p^{\prime}}^{\prime}$-algebra with dimension equal to $l$ and $\operatorname{deg}_{A_{p^{\prime}}^{\prime}}\left(A_{p^{\prime}}^{\prime} \otimes_{A^{\prime}} G^{\prime}\right)=\operatorname{deg}_{A_{p}}\left(A_{p} \otimes_{A} G\right)=r$ (see the argument before Proposition 4.6). By [6, 7.3], $\operatorname{dim} A_{p^{\prime}}^{\prime} \otimes_{A^{\prime}} A^{\prime \prime}=\operatorname{dim} A_{p^{\prime}}^{\prime} \otimes_{A^{\prime}} G^{\prime}=l=d-s$. Hence $\operatorname{dim} A^{\prime \prime}=\operatorname{dim} G^{\prime}=d$. Notice for every minimal prime ideal $p^{\prime \prime}$ of $A^{\prime \prime}$ with $\operatorname{dim} A^{\prime \prime} / p^{\prime \prime}=d, p^{\prime \prime}$ is the extension $p^{\prime} A^{\prime \prime}$, where $p^{\prime}$ is a prime ideal of $A^{\prime}$ with $\operatorname{dim} A^{\prime} / p^{\prime}=s$. Thus $A_{p^{\prime \prime}}^{\prime \prime}$ is reduced and $\operatorname{rank}_{A_{p^{\prime \prime}}^{\prime \prime}} G_{p^{\prime \prime}}^{\prime}=\operatorname{rank}_{A_{p^{\prime}}^{\prime} \otimes_{A^{\prime}} A^{\prime \prime}} A_{p^{\prime}}^{\prime} \otimes_{A^{\prime}} G^{\prime}=$ $\operatorname{deg}_{A_{p^{\prime}}^{\prime}}\left(A_{p^{\prime}}^{\prime} \otimes_{A^{\prime}} G^{\prime}\right)=r$ (see also the argument before Proposition 4.6).

Now consider the ideal $I=\left(G_{01}, y_{1}^{\prime}, \ldots, y_{l}^{\prime}\right) G^{\prime}$. Let

$$
y_{\alpha}^{\prime \prime}=\sum_{\beta=1}^{n} w_{\alpha \beta} a_{\beta} T+\sum_{\beta=n+1}^{n+l} w_{\alpha \beta} y_{\beta-n}^{\prime}, \quad 1 \leq \alpha \leq l,
$$

$x_{i}^{\prime}=\sum_{j=1}^{n} z_{i j} a_{j} T, 1 \leq i \leq s$; then $y_{1}^{\prime \prime}, \ldots, y_{l}^{\prime \prime}, x_{1}^{\prime}, \ldots, x_{s}^{\prime}$ form a super-reduction for $I$. Indeed, it is easy to see that $y_{1}^{\prime \prime}, \ldots, y_{l}^{\prime \prime}$ are filter-regular with respect to $I$, as they are generic linear combinations of the generators of $I$. Moreover, for every prime ideal $P^{\prime} \in \operatorname{Spec}\left(G^{\prime}\right)$, if $P^{\prime}$ contains $\left(y_{1}^{\prime \prime}, \ldots, y_{l}^{\prime \prime}\right)$ and $G_{01}$, then $P^{\prime}$ contains $\sum_{\beta=n+1}^{n+l} w_{\alpha \beta} y_{\beta-n}^{\prime}$ for $1 \leq \alpha \leq l$. Since the matrix $\left(w_{\alpha \beta}\right), 1 \leq \alpha \leq l, n+1 \leq \beta \leq$ $n+l$, is invertible over $G^{\prime}, P^{\prime}$ contains $y_{\mu}^{\prime}$ for $1 \leq \mu \leq l$. Thus $P^{\prime} \supseteq I$. This shows 
that $y_{1}^{\prime \prime}, \ldots, y_{l}^{\prime \prime}, x_{1}^{\prime}, \ldots, x_{s}^{\prime}$ form a filter-regular sequence for $G^{\prime}$ with respect to $I$. By a similar argument, one can actually show that they form a super-reduction for $I$. Let $\mathfrak{P}^{\prime}=\left\{P^{\prime} \in \operatorname{Min}\left(y_{1}^{\prime \prime}, \ldots, y_{l}^{\prime \prime}, x_{1}^{\prime}, \ldots, x_{s-1}^{\prime}\right) \mid \operatorname{dim} G^{\prime} / P^{\prime}=1, G_{01} G^{\prime} \nsubseteq P^{\prime}\right\}$. Notice that $y_{1}^{\prime \prime}, \ldots, y_{l}^{\prime \prime}$ are generic linear combinations of the generators of $\left(G_{10}, G_{01}\right) G^{\prime}$. By the proof of Theorems 3.1, 4.1 and the argument similar to [17, 6.5], we have

$$
\begin{aligned}
r e\left(A^{\prime \prime}\right) & =j_{d}(I) \\
= & \lambda\left(G^{\prime} /\left(\left(y_{1}^{\prime \prime}, \ldots, y_{l}^{\prime \prime}, x_{1}^{\prime}, \ldots, x_{s-1}^{\prime}\right) G^{\prime}:_{G^{\prime}}\langle I\rangle+x_{s}^{\prime} G^{\prime}\right)\right) \\
= & e\left(G^{\prime} /\left(y_{1}^{\prime \prime}, \ldots, y_{l}^{\prime \prime}, x_{1}^{\prime}, \ldots, x_{s-1}^{\prime}\right) G^{\prime}:_{G^{\prime}}\langle I\rangle\right) \\
= & \sum_{P^{\prime} \in \mathfrak{P}^{\prime}} \lambda\left(G_{P^{\prime}}^{\prime} /\left(y_{1}^{\prime \prime}, \ldots, y_{l}^{\prime \prime}, x_{1}^{\prime}, \ldots, x_{s-1}^{\prime}\right) G_{P^{\prime}}^{\prime}\right) e\left(G^{\prime} / P^{\prime}\right) \\
= & e\left(G^{\prime} /\left(\left(x_{1}^{\prime}, \ldots, x_{s-1}^{\prime}\right) G^{\prime}:_{G^{\prime}}\left\langle G_{01} G^{\prime}\right\rangle+\left(y_{1}^{\prime \prime}, \ldots, y_{l}^{\prime \prime}\right)\right)\right) \\
= & e\left(G^{\prime} /\left(x_{1}^{\prime}, \ldots, x_{s-1}^{\prime}\right) G^{\prime}:_{G^{\prime}}\left\langle G_{01} G^{\prime}\right\rangle\right) \\
= & e\left(G^{\prime} /\left(\left(x_{1}^{\prime}, \ldots, x_{s-1}^{\prime}\right) G^{\prime}:_{G^{\prime}}\left\langle G_{01} G^{\prime}\right\rangle+x_{s}^{\prime} G^{\prime}\right)\right) \\
= & \operatorname{deg} v_{s}\left(x_{1}^{\prime}, \ldots, x_{s}^{\prime}, G^{\prime}\right) \\
= & c_{d-s}\left(A_{1} B^{\prime}\right)=c_{d-s}\left(A_{1} B\right) .
\end{aligned}
$$

Finally since $e\left(A^{\prime \prime}\right)=e\left(A^{\prime}\right)=e(A)$, we are done.

Remark 4.7. In Proposition 4.6, if in addition we assume that $B$ is equidimensional and universally catenary, then $G$ is also equidimensional by [17, 2.2]. Observe for every $p \in \mathfrak{P}$, there exists $P \in \operatorname{Min}(G)$ which contracts back to $p$. Since $G$ is equidimensional, for every such $P, \operatorname{dim} G / P=d$. Notice $\operatorname{dim} A / p=s$. Hence Quot $(G / P)$ has transcendence degree $l=d-s$ over $\operatorname{Quot}(A / p)$. Therefore $\operatorname{dim} A_{p} \otimes_{A} G=l$ for every prime ideal $p \in \mathfrak{P}$.

Remark 4.8. First observe in Proposition 4.6, for each $p \in \mathfrak{P}$, since $A_{p} \otimes_{A} G$ is standard graded over $A_{p}, r=\operatorname{deg}_{A_{p}}\left(A_{p} \otimes_{A} G\right)=e\left(A_{p} \otimes_{A} G\right.$ ) (see the argument before Proposition 4.6). Moreover if we assume $A$ is a domain, one can replace $r$ by $\operatorname{rank}_{A\left[y_{1}, \ldots, y_{l}\right]} B$, where $y_{i}=\sum_{j=1}^{\tau} \lambda_{i j} b_{j}, 1 \leq i \leq l$, for general elements $\left(\lambda_{i j}\right) \in k^{l \tau}$, and $k$ is the residue field of $A$.

Proof. One can show $\left(\operatorname{rank}_{A^{\prime}\left[y_{1}^{\prime}, \ldots, y_{l}^{\prime}\right]} B^{\prime}\right) \cdot e(A)=c_{d-s}\left(A_{1} B\right)$ by the same argument as in Proposition 4.6, where $A^{\prime}, B^{\prime}, y_{i}^{\prime}, 1 \leq i \leq l$, are constructed in the same way as in that proof. By [17, p.251], $\operatorname{rank}_{A^{\prime}\left[y_{1}^{\prime}, \ldots, y_{l}^{\prime}\right]} B^{\prime}=\operatorname{rank}_{A\left[y_{1}, \ldots, y_{l}\right]} B$ for general elements $\left(\lambda_{i j}\right) \in k^{l \tau}$. Since $\operatorname{re}(A)=c_{d-s}\left(A_{1} B\right)$, we are done.

By Theorem 3.1 and Proposition 4.6, we have the following theorem.

Theorem 4.9. In the same setting as Proposition 4.6, let $g=\mathrm{ht} A_{1} B$. For general elements $\Lambda=\left(\lambda_{i j}\right) \in R_{0}^{s n}$, define $x_{i}$ and $\mathfrak{Q}_{i}, 0 \leq i \leq s$, as before. Then

$$
\begin{aligned}
e(B)=r e(A) & +\sum_{q \in \mathfrak{Q}_{0}} e_{A_{1} B_{q}}\left(B_{q}\right) e(B / q) \\
& +\sum_{i=\max \{1, g\}}^{s-1} \sum_{q \in \mathfrak{Q}_{i}} j_{1}\left(\frac{A_{1} B_{q}}{\left(x_{1}, \ldots, x_{i-1}\right) B_{q}}\right) e(B / q) .
\end{aligned}
$$

By Corollaries 3.3, 3.5 and Proposition 4.6, we have the following corollary. 
Corollary 4.10. Let $A \subseteq B$ be as in Proposition 4.6. Assume $B$ is CohenMacaulay. Also write $A_{1} A=\left(a_{1}, \ldots, a_{n}\right) A$, where $a_{1}, \ldots, a_{n}$ are homogeneous elements of degree one, and $g=\operatorname{ht} A_{1} B$. Let $\mathfrak{P}=\{p \in \operatorname{Spec}(A) \mid \operatorname{dim} A / p=\operatorname{dim} A\}$ and assume that $A_{p}$ is reduced and $\operatorname{deg}_{A_{p}}\left(A_{p} \otimes_{A} G\right)=r$ for every prime ideal $p \in \mathfrak{P}$, where $G=\operatorname{gr}_{A_{1} B}(B)$. For general elements $\Lambda=\left(\lambda_{i j}\right) \in R_{0}^{s n}$, define $x_{i}, H_{i-1}$ and $\Lambda_{i-1}, 1 \leq i \leq s$, as before.

(a) Assume the ideal $A_{1} B$ satisfies condition $G_{s}$ and the Artin-Nagata property $A N_{s-2}^{-}$. Then

$$
e(B)=r e(A)+e\left(B / A_{1} B\right)+\sum_{i=g+1}^{s-1} e\left(B /\left(H_{i-1}+A_{1} B\right)\right) .
$$

(b) Assume $A_{1} B_{q}$ is a complete intersection for every $q \in \operatorname{Proj}(B)$. Then

$$
e(B)=r e(A)+e\left(B / A_{1} B\right)+\sum_{i=g+1}^{s-1} e\left(B /\left(F_{i-1}+A_{1} B\right)\right),
$$

where $F_{i-1}=\operatorname{Fitt}_{0}\left(A_{1} B /\left(x_{1}, \ldots, x_{i-1}\right) B\right)$.

(c) Assume $A_{1} B$ is a perfect ideal of height 2 which satisfies condition $G_{s}$. Write $\mu\left(A_{1} B\right)=n$ and let $X_{n \times(n-1)}$ be a matrix such that $A_{1} B=I_{n-1}(X)$. Then

$$
e(B)=r e(A)+e\left(B / A_{1} B\right)+\sum_{i=3}^{s-1} e\left(B /\left(I_{n}\left(X \mid \Lambda_{i-1}^{T}\right)+A_{1} B\right)\right) .
$$

(d) Assume $A_{1} B$ is a perfect Gorenstein ideal of height 3 which satisfies condition $G_{s}$. Write $\mu\left(A_{1} B\right)=n$ and let $X_{n \times n}=\left(x_{i j}\right)$ be an alternating matrix such that $A_{1} B=\operatorname{Pf}_{n-1}(X)$. Let $T_{i-1}=\left(\begin{array}{cc}X & \Lambda_{i-1}^{T} \\ -\Lambda_{i-1} & 0\end{array}\right)$ and $J\left(T_{i-1}\right)$ be the B-ideal generated by the Pfaffians of all principal submatrices of $T_{i-1}$ which contain $X$ for $4 \leq i \leq$ $s-1$. Then

$$
e(B)=r e(A)+e\left(B / A_{1} B\right)+\sum_{i=4}^{s-1} e\left(B /\left(J\left(T_{i-1}\right)+A_{1} B\right)\right) .
$$

Proof. Since $B$ is Cohen-Macaulay, it is equidimensional and universally catenary. By Remark $4.7, \operatorname{dim} A_{p} \otimes_{A} G=d-s$ for every prime ideal $p \in \mathfrak{P}$. We are done by Corollaries 3.3 and 3.5 and Proposition 4.6.

\section{Applications to the Special Fiber Ring}

In this section, we are going to apply our formulas to the special fiber ring $k \otimes_{R} \mathscr{R}(I)$, where $I \subseteq R$ is an ideal generated by forms of the same degree in a standard graded $k$-algebra $R$. We have the following theorem:

Theorem 5.1. Let $R=k\left[R_{1}\right]$ be a reduced standard graded Noetherian ring of dimension $d$ over an infinite field $k$. Let $I=\left(a_{1}, \ldots, a_{n}\right) R$ be an $R$-ideal of height $g>0$, where $a_{1}, \ldots, a_{n}$ are homogeneous elements of degree $\delta>0$. Write $s=\ell(I)$, $k\left[I_{\delta}\right]$ the $k$-algebra generated by the forms in $I$ of degree $\delta, R^{(\delta)}$ the $\delta$-th Veronese subring of $R$ and $G=\operatorname{gr}_{I_{\delta} R^{(\delta)}}\left(R^{(\delta)}\right)$. Let $\mathfrak{P}=\left\{p \in \operatorname{Spec}\left(k\left[I_{\delta}\right]\right) \mid \operatorname{dim} k\left[I_{\delta}\right] / p=\right.$ $\left.\operatorname{dim} k\left[I_{\delta}\right]\right\}$. For every prime ideal $p \in \mathfrak{P}$, assume $\operatorname{dim} k\left[I_{\delta}\right]_{p} \otimes_{k\left[I_{\delta}\right]} G=d-s$ and $\operatorname{deg}_{k\left[I_{\delta}\right]_{p}}\left(k\left[I_{\delta}\right]_{p} \otimes_{k\left[I_{\delta}\right]} G\right)=r$ (notice when $\left.s=d, r=\operatorname{rank}_{k\left[I_{\delta}\right]_{p}}\left(R^{(\delta)}\right)_{p}\right)$. For general 
elements $\Lambda=\left(\lambda_{i j}\right) \in k^{s n}$, let $x_{i}=\sum_{j=1}^{n} \lambda_{i j} a_{j}, J_{i-1}=\left(x_{1}, \ldots, x_{i-1}\right) R:_{R}\langle I\rangle$ and $\mathfrak{Q}_{i}=\left\{q \in \operatorname{Min}\left(J_{i-1}+I\right) \mid \operatorname{dim} R / q=d-i\right\}$ for $1 \leq i \leq s$. Then

$$
e\left(k\left[I_{\delta}\right]\right)=e(R) \frac{\delta^{d-1}}{r}-\sum_{i=g}^{s-1} \sum_{q \in \mathfrak{Q}_{i}} j_{1}\left(\frac{I_{q}}{\left(x_{1}, \ldots, x_{i-1}\right)_{q}}\right) e(R / q) \frac{\delta^{d-i-1}}{r} .
$$

Proof. Let $J_{i-1}^{(\delta)}=\left(x_{1}, \ldots, x_{i-1}\right) R^{(\delta)}:_{R^{(\delta)}}\left\langle I_{\delta} R^{(\delta)}\right\rangle, 1 \leq i \leq s$. For every $q \in$ $\operatorname{Proj}(R)$, let $q^{(\delta)}=q \cap R^{(\delta)}$. Then $R_{q^{(\delta)}}^{(\delta)} \subseteq R_{q}$ is an étale local extension [17]. By flatness we have $J_{i-1}^{(\delta)} R_{q}=J_{i-1} R_{q}$. Since there are natural isomorphisms from $\operatorname{Proj}(R)$ to $\operatorname{Proj}\left(R^{(\delta)}\right)$ and from $\operatorname{Proj}(R / I)$ to $\operatorname{Proj}\left((R / I)^{(\delta)}\right)=\operatorname{Proj}\left(R^{(\delta)} / I_{\delta} R^{(\delta)}\right)$, they induce a one-to-one correspondence between the set $\mathfrak{Q}_{i}$ and the set of all primes $q^{(\delta)}$ in $V\left(J_{i-1}^{(\delta)}+I_{\delta} R^{(\delta)}\right)$ with $\operatorname{dim} R^{(\delta)} / q^{(\delta)}=d-i$ for each $i$ with $1 \leq i \leq s$. Let $\bar{R}^{(\delta)}=R^{(\delta)} /\left(x_{1}, \ldots, x_{i-1}\right) R^{(\delta)}, \bar{R}=R /\left(x_{1}, \ldots, x_{i-1}\right) R$. Since $\bar{q}^{(\delta)} \bar{R}_{\bar{q}}=\bar{q} \bar{R}_{\bar{q}}$, by flatness we have

$$
\lambda_{\bar{R}_{\bar{q}^{(\delta)}}^{(\delta)}}\left(\Gamma_{\bar{q}^{(\delta)} \bar{R}_{\bar{q}^{(\delta)}}^{(\delta)}}\left(\bar{I}_{\delta}^{j} \bar{R}_{\bar{q}^{(\delta)}}^{(\delta)} / \bar{I}_{\delta}^{j+1} \bar{R}_{\bar{q}^{(\delta)}}^{(\delta)}\right)\right)=\lambda_{\bar{R}_{\bar{q}}}\left(\Gamma_{\bar{q} \bar{R}_{\bar{q}}}\left(\bar{I}^{j} \bar{R}_{\bar{q}} / \bar{I}^{j+1} \bar{R}_{\bar{q}}\right)\right)
$$

for every $j \geq 0$. Thus we have $j_{1}\left(I_{\delta} \bar{R}_{\bar{q}^{(\delta)}}^{(\delta)}\right)=j_{1}\left(I \bar{R}_{\bar{q}}\right)$. Also recall $e\left(R^{(\delta)}\right)=$ $e(R) \delta^{d-1}$ and $e\left(R^{(\delta)} /\left(J_{i-1}^{(\delta)}+I_{\delta} R^{(\delta)}\right)\right)=e\left(R /\left(J_{i-1}+I\right)\right) \delta^{d-i-1}$.

We rescale the grading of both $k\left[I_{\delta}\right]$ and $R^{(\delta)}$ so that $k\left[I_{\delta}\right] \subseteq R^{(\delta)}$ is a homogeneous inclusion of standard graded Noetherian algebras. Notice $k\left[I_{\delta}\right] \simeq k \otimes_{R} \mathscr{R}(I)$, $\operatorname{dim} k\left[I_{\delta}\right]=\ell(I)=s$ and $\operatorname{dim} R^{(\delta)}=d$. Thus we apply Theorems 4.1 and 4.9 to the homogeneous inclusion $k\left[I_{\delta}\right] \subseteq R^{(\delta)}$ to get equation (8).

By Theorem 5.1 and the proof of Corollaries 3.3 and 3.5, we have the following corollary:

Corollary 5.2. Let $R$ and $I$ be as in Theorem 5.1. Assume $R$ is Cohen-Macaulay. Also, let $\mathfrak{P}=\left\{p \in \operatorname{Spec}\left(k\left[I_{\delta}\right]\right) \mid \operatorname{dim} k\left[I_{\delta}\right] / p=\operatorname{dim} k\left[I_{\delta}\right]\right\}$. For every prime ideal $p \in$ $\mathfrak{P}$, assume $\operatorname{deg}_{k\left[I_{\delta}\right]_{p}}\left(k\left[I_{\delta}\right]_{p} \otimes_{k\left[I_{\delta}\right]} G\right)=r\left(\right.$ notice when $\left.s=d, r=\operatorname{rank}_{k\left[I_{\delta}\right]_{p}}\left(R^{(\delta)}\right)_{p}\right)$, where $k\left[I_{\delta}\right], R^{(\delta)}$ and $G$ are defined as in Theorem 5.1. For general elements $\Lambda=$ $\left(\lambda_{i j}\right) \in k^{s n}$, let $x_{i}=\sum_{j=1}^{n} \lambda_{i j} a_{j}, H_{i-1}=\left(x_{1}, \ldots, x_{i-1}\right) R:_{R} I$ and $\Lambda_{i-1}$ be the submatrix consisting of the first $i-1$ rows of $\Lambda$ for $1 \leq i \leq s$.

(a) Assume the ideal I satisfies condition $G_{s}$ and the Artin-Nagata property $A N_{s-2}^{-}$. Then

$$
e\left(k\left[I_{\delta}\right]\right)=e(R) \frac{\delta^{d-1}}{r}-e(R / I) \frac{\delta^{d-g-1}}{r}-\sum_{i=g+1}^{s-1} e\left(R /\left(H_{i-1}+I\right)\right) \frac{\delta^{d-i-1}}{r} .
$$

(b) Assume $I_{q}$ is a complete intersection for every $q \in \operatorname{Proj}(R)$. Then

$$
e\left(k\left[I_{\delta}\right]\right)=e(R) \frac{\delta^{d-1}}{r}-e(R / I) \frac{\delta^{d-g-1}}{r}-\sum_{i=g+1}^{s-1} e\left(R /\left(F_{i-1}+I\right)\right) \frac{\delta^{d-i-1}}{r},
$$

where $F_{i-1}=\operatorname{Fitt}_{0}\left(I /\left(x_{1}, \ldots, x_{i-1}\right) R\right)$. 
(c) Assume $I$ is a perfect ideal of height 2 which satisfies condition $G_{s}$. Write $\mu(I)=n$ and let $X_{n \times(n-1)}$ be a matrix such that $I=I_{n-1}(X)$. Then

$$
e\left(k\left[I_{\delta}\right]\right)=e(R) \frac{\delta^{d-1}}{r}-e(R / I) \frac{\delta^{d-3}}{r}-\sum_{i=3}^{s-1} e\left(R /\left(I_{n}\left(X \mid \Lambda_{i-1}^{T}\right)+I\right)\right) \frac{\delta^{d-i-1}}{r} .
$$

(d) Assume $I$ is a perfect Gorenstein ideal of height 3 which satisfies condition $G_{s}$. Write $\mu(I)=n$, and let $X_{n \times n}$ be an alternating matrix such that $I=$ $\operatorname{Pf}_{n-1}(X)$. Let $T_{i-1}=\left(\begin{array}{cc}X & \Lambda_{i-1}^{T} \\ -\Lambda_{i-1} & 0\end{array}\right)$ and $J\left(T_{i-1}\right)$ be the $R$-ideal generated by the Pfaffians of all principal submatrices of $T_{i-1}$ which contain $X$ for $4 \leq i \leq s-1$. Then

$$
e\left(k\left[I_{\delta}\right]\right)=e(R) \frac{\delta^{d-1}}{r}-e(R / I) \frac{\delta^{d-4}}{r}-\sum_{i=4}^{s-1} e\left(R /\left(J\left(T_{i-1}\right)+I\right)\right) \frac{\delta^{d-i-1}}{r} .
$$

Now we are going to apply Theorem 5.1 and Corollary 5.2 to the following cases.

Application 1. If char $k=0$ and $R$ is a domain, Theorem 5.1 yields an upper bound for the reduction number $r_{J}(I)$ of $I$ with respect to any reduction $J$, because $r_{J}(I) \leq e\left(k\left[I_{\delta}\right]\right)$ by [22]. This gives a sharper bound than that of [17].

Application 2. The ideal $I=\left(a_{1}, \ldots, a_{n}\right) R$ of Theorem 5.1 induces a rational map $\phi: \operatorname{Proj}(R) \rightarrow \mathbb{P}_{k}^{n-1}$ defined by $\phi(p)=\left(a_{1}(p), \ldots, a_{n}(p)\right)$, where $p \in \operatorname{Proj}(R) \backslash V(I)$ and $a_{i}(p)$ is $a_{i}$ evaluated at $p$. Let $\operatorname{im}(\phi)$ be the image of $\phi$. Then Theorem 5.1 provides a formula for the degree of $\operatorname{im}(\phi)$. Indeed the homogeneous coordinate ring of $\operatorname{im}(\phi)$ is the special fiber ring $k\left[I_{\delta}\right]$. Thus $\operatorname{deg}(\operatorname{im}(\phi))=e\left(k\left[I_{\delta}\right]\right)$.

Application 3 (Generalized Teissier's Plücker formula). Let $X \subseteq \mathbb{P}_{k}^{n}$ be a hypersurface defined by a homogeneous irreducible polynomial $f$ of degree $\delta>0$ over an algebraically closed field $k$. Let $X^{\prime}$ be the dual variety of $X$. Notice that in this case $X^{\prime}$ is the image of the Gauss map of $X$. Set $R=k\left[Y_{0}, \ldots, Y_{n}\right] /(f)$ and $I=\left(\partial f / \partial Y_{0}, \ldots, \partial f / \partial Y_{n}\right) R$ (the Jacobian ideal of $R$ ). Then $\operatorname{dim} R=n$ and $I$ is generated by homogeneous elements of degree $\delta-1$. Since $A\left(X^{\prime}\right) \simeq k\left[I_{\delta-1}\right]$, we can apply Theorem 5.1 and Corollary 5.2 to $R$ to get the degree $\delta^{\prime}$ of $X^{\prime}$.

Let $s=\ell(I)$ and $g=$ ht $I$. For general elements $\left(\lambda_{i j}\right) \in k^{s(n+1)}$, let $x_{i}=$ $\sum_{j=0}^{n} \lambda_{i j} \partial f / \partial Y_{j}$ and $I_{i-1}=\left(x_{1}, \ldots, x_{i-1}\right) R$ for $1 \leq i \leq s$. Now for each $i$, decompose $V\left(I_{i-1}\right)$ into a union of irreducible components $V_{i-1}^{1}, \ldots, V_{i-1}^{b}$, where $V_{i-1}^{j} \nsubseteq \operatorname{Sing}(X)$ for $1 \leq j \leq a$ and $V_{i-1}^{j} \subseteq \operatorname{Sing}(X)$ for $a+1 \leq j \leq b$. Define $\mathfrak{Q}_{i}$ to be the set of irreducible components of $\left(V_{i-1}^{1} \cup \cdots \cup V_{i-1}^{a}\right) \cap \operatorname{Sing}(X)$ with dimension $n-i-1$.

Let $K=\operatorname{Quot}\left(k\left[I_{\delta-1}\right]\right), G=\operatorname{gr}_{\left(I_{\delta-1}\right) R^{(\delta-1)}}\left(R^{(\delta-1)}\right)$ and $r=\operatorname{deg}_{K}\left(K \otimes_{k\left[I_{\delta-1}\right]} G\right)$. Observe that if the dual variety $X^{\prime}$ is again a hypersurface, i.e., $\ell(I)=\operatorname{dim} R=n$, $r$ is just the degree of the Gauss map.

We have

$$
\delta^{\prime}=\frac{\delta(\delta-1)^{n-1}}{r}-\sum_{i=g}^{s-1} \sum_{q \in \mathfrak{Q}_{i}} j_{1}\left(I_{q} /\left(I_{i-1}\right)_{q}\right) \operatorname{deg}(q) \frac{(\delta-1)^{n-i-1}}{r} .
$$

This formula holds for a hypersurface with arbitrary dual variety and singularities. It also gives a generalization of Teissier's Plücker formula. 
Moreover, letting $H_{i-1}=\left(x_{1}, \ldots, x_{i-1}\right) R:_{R} I$ and $\Lambda_{i-1}$ be the submatrix consisting of the first $i-1$ rows of $\Lambda=\left(\lambda_{i j}\right)$ for $1 \leq i \leq s$, we have the following cases:

(a) Assume $I$ satisfies condition $G_{s}$ and the Artin-Nagata property $A N_{s-2}^{-}$. Then

$$
\begin{aligned}
\delta^{\prime}= & \frac{\delta(\delta-1)^{n-1}}{r}-\operatorname{deg}(V(I)) \frac{(\delta-1)^{n-g-1}}{r} \\
& -\sum_{i=g+1}^{s-1} \operatorname{deg}\left(V\left(H_{i-1}+I\right)\right) \frac{(\delta-1)^{n-i-1}}{r} .
\end{aligned}
$$

(b) Assume $I$ is a complete intersection on $X$. Then

$$
\begin{aligned}
\delta^{\prime}= & \frac{\delta(\delta-1)^{n-1}}{r}-\operatorname{deg}(V(I)) \frac{(\delta-1)^{n-g-1}}{r} \\
& -\sum_{i=g+1}^{s-1} \operatorname{deg}\left(V\left(F_{i-1}+I\right)\right) \frac{(\delta-1)^{n-i-1}}{r},
\end{aligned}
$$

where $F_{i-1}=\operatorname{Fitt}_{0}\left(I / I_{i-1}\right)$.

(c) Assume $I$ is a perfect ideal of height 2 which satisfies condition $G_{s}$. Write $\mu(I)=n$, and let $W_{n \times(n-1)}$ be a matrix such that $I=I_{n-1}(W)$. Then

$$
\begin{aligned}
\delta^{\prime}= & \frac{\delta(\delta-1)^{n-1}}{r}-\operatorname{deg}(V(I)) \frac{(\delta-1)^{n-3}}{r} \\
& -\sum_{i=3}^{s-1} \operatorname{deg}\left(V\left(I_{n}\left(W \mid \Lambda_{i-1}^{T}\right)+I\right)\right) \frac{(\delta-1)^{n-i-1}}{r} .
\end{aligned}
$$

(d) Assume $I$ is a perfect Gorenstein ideal of height 3 which satisfies condition $G_{s}$. Write $\mu(I)=n$, and let $W_{n \times n}$ be an alternating matrix such that $I=\operatorname{Pf}_{n-1}(W)$. Let $T_{i-1}=\left(\begin{array}{cc}W & \Lambda_{i-1}^{T} \\ -\Lambda_{i-1} & 0\end{array}\right)$ and $J\left(T_{i-1}\right)$ be the $R$-ideal generated by the Pfaffians of all principal submatrices of $T_{i-1}$ which contain $W$. Then

$$
\begin{aligned}
\delta^{\prime}= & \frac{\delta(\delta-1)^{n-1}}{r}-\operatorname{deg}(V(I)) \frac{(\delta-1)^{n-4}}{r} \\
& -\sum_{i=4}^{s-1} \operatorname{deg}\left(V\left(J\left(T_{i-1}\right)+I\right)\right) \frac{(\delta-1)^{n-i-1}}{r} .
\end{aligned}
$$

We will finish the paper by giving the following example.

Example. Surfaces in 3-Space (see [4, 9.3.7] or [19]). Let $X: y_{1}^{2} y_{3}-y_{2}^{2} y_{0}=0$ be a hypersurface of degree 3 in $\mathbb{P}_{k}^{3}$, where $k$ is an algebraically closed field. Observe

$$
R=k\left[y_{0}, y_{1}, y_{2}, y_{3}\right] /\left(y_{1}^{2} y_{3}-y_{2}^{2} y_{0}\right)=k\left[\bar{y}_{0}, \bar{y}_{1}, \bar{y}_{2}, \bar{y}_{3}\right]
$$

is the homogeneous coordinate ring of $X$ and $I=V\left(\bar{y}_{2}^{2}, \bar{y}_{1} \bar{y}_{3}, \bar{y}_{2} \bar{y}_{0}, \bar{y}_{1}^{2}\right)$ is the Jacobian ideal of $R$. The singularity locus $\operatorname{Sing}(X)$ of $X$ is equal to $V(I)$ which is a line consisting of double points and two pinch points: $(0,0,0,1),(1,0,0,0)$. Since the special fiber ring $F(I)=k\left[\bar{y}_{2}^{2}, \bar{y}_{1} \bar{y}_{3}, \bar{y}_{2} \bar{y}_{0}, \bar{y}_{1}^{2}\right]$ is the homogeneous coordinate ring of the dual variety $X^{\prime}$, we can use our formula to find the class $\delta^{\prime}=\operatorname{degree}\left(X^{\prime}\right)$ of the variety $X$.

Observe $\operatorname{dim} R=3, I$ is generated by forms of the same degree 2 , ht $I=1$, $\ell(I)=3$, and $r=1$ the degree of the Gauss map. Let $x_{1}=\bar{y}_{1} \bar{y}_{3}-\bar{y}_{2} \bar{y}_{0}, x_{2}=$ 
$\bar{y}_{1}^{2}-\bar{y}_{2}^{2}+\bar{y}_{1} \bar{y}_{3}$, then

$$
\mathfrak{Q}_{1}=\left\{\left(\bar{y}_{1}, \bar{y}_{2}\right)\right\}, \mathfrak{Q}_{2}=\left\{\left(\bar{y}_{0}, \bar{y}_{1}, \bar{y}_{2}\right),\left(\bar{y}_{1}, \bar{y}_{2}, \bar{y}_{3}\right),\left(\bar{y}_{0}-\bar{y}_{3}, \bar{y}_{1}, \bar{y}_{2}\right)\right\},
$$

and

$$
\begin{aligned}
\delta^{\prime} & =\operatorname{degree}\left(X^{\prime}\right)=e(F(I)) \\
& =3 \cdot 2^{2}-2 \cdot \sum_{q \in \mathfrak{Q}_{1}} j_{1}\left(I_{q}\right)-\sum_{q \in \mathfrak{Q}_{2}} j_{1}\left(I_{q} /\left(\bar{y}_{1} \bar{y}_{3}-\bar{y}_{2} \bar{y}_{0}\right)_{q}\right) \\
& =3 \cdot 2^{2}-2 \cdot 2-(2+2+1)=3 .
\end{aligned}
$$

Indeed, $X^{\prime}$ is a hypersurface which is isomorphic to $X$.

\section{ACKNOWLEDGMent}

The author is very grateful to the referee for a meticulous reading and detailed recommendations which led to the improvements in the text.

\section{REFERENCES}

[1] R. Achilles and M. Manaresi, Multiplicity for ideals of maximal analytic spread and intersection theory, J. Math. Kyoto Univ. 33 (1993), 1029-1046. MR1251213 (94m:13030)

[2] R. Achilles and M. Manaresi, Multiplicities of a bigraded ring and intersection theory, Math. Ann. 309 (1997), 573-591. MR1483824 (99a:14005)

[3] R. Achilles and M. Manaresi, Generalized Samuel multiplicities and applications, Rend. Sem. Mat. Univ. Pol. Torino. 64 (2006), 345-372. MR.2295444 (2007m:13031)

[4] W. Fulton, Intersection theory, Springer-Verlag, Berlin, 1998. MR.1644323 (99d:14003)

[5] H. Flenner and M. Manaresi, A numerical characterization of reduction ideals, Math. Z. 238 (2001), 205-214. MR1860742 (2002h:13034)

[6] H. Flenner, B. Ulrich and W. Vogel, On limits of joins of maximal dimension, Math. Ann. 308 (1997), 291-318. MR.1464904 (98h:13013)

[7] R. Hartshorne, C. Huneke and B. Ulrich, Residual intersections of licci ideals are glicci, preprint.

[8] C. Huneke, Strongly Cohen-Macaulay schemes and residual intersections, Trans. Amer. Math. Soc. 277 (1983), 739-763. MR694386 (84m:13023)

[9] C. Huneke and B. Ulrich, Residual intersections, J. Reine Angew. Math. 390 (1988), 1-20. MR953673 (89j:13024)

[10] C. Huneke and B. Ulrich, Generic residual intersections, Commutative algebra (Salvador, 1988), Lecture Notes in Math. 1430, Springer, Berlin, 1990, 47-60. MR1068323(91h:13013)

[11] S. L. Kleiman, A generalized Teissier-Plücker formula, Contemp. Math. 162 (1994), 249-260. MR1272702 (95c:14073)

[12] M. Kline, Mathematical thought from ancient to modern times, Oxford Univ. Press, 1972. $\operatorname{MR} 0472307$ (57:12010)

[13] A. Kustin and B. Ulrich, A family of complexes associated to an almost alternating map; with applications to residual intersection, Memoirs AMS 95 (1992), no 461. MR.1091668 (92i:13012)

[14] D.G. Northcott and D. Rees, Reductions of ideals in local rings, Proc. Cambridge Philos. Soc. 50 (1953), 145-158. MR0059889 (15:596a)

[15] P. Samuel, La notion de multiplicité en algèbre et en géométrie algébrique, J. Math. Pures Appl. 30 (1951), 159-274. MR0048103 (13:980c)

[16] J-P. Serre, Algèbre locale. Multiplicités, Springer-Verlag, Berlin-New York, 1965. MR 0201468 $(34: 1352)$

[17] A. Simis, B. Ulrich and W. V. Vasconcelos, Codimension, multiplicity and integral extensions, Math. Proc. Camb. Phil. Soc. 130 (2001), 237-257. MR1806775 (2002c:13017)

[18] B. Teissier, Sur diverses conditions numériques d'équisingularité des familles de courbes, Centre de Math. École Polytechnique, 1975. 
[19] A. Thorup, Generalized Plücker formulas, Recent progress in intersection theory (Bologna, 1997), Trends Math. Birkhäuser Boston, Boston, MA, 2000, 299-327. MR1849300 (2002h:14007)

[20] B. Ulrich, Artin-Nagata properties and reductions of ideals, Commutative algebra: syzygies, multiplicities, and birational algebra (South Hadley, MA, 1992), Contemp. Math. 159, Amer. Math. Soc. Providence, RI, 1994, 373-400. MR.1266194 (95a:13017)

[21] J. Validashti, Multiplicities of graded algebras, Ph.D. thesis, Purdue University, 2007.

[22] W.V. Vasconcelos, The reduction number of an algebra, Compositio Math. 104 (1996), 189197. MR.1421399 (98f:13001)

Department of Mathematics, The University of Notre Dame, South Bend, Indiana 46556

E-mail address: yxie@nd.edu 